\title{
Mathematical and numerical models for the cardiac electromechanical function
}

\author{
L. Dedè ${ }^{1}$, A. Quarteroni ${ }^{1,2}$, and F. Regazzoni ${ }^{1, *}$

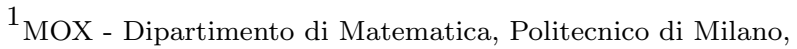 \\ P.zza Leonardo da Vinci 32, 20133 Milano, Italy \\ ${ }^{2}$ Mathematics Institute, École Polytechnique Fédérale de Lausanne (EPFL), \\ Av. Piccard, CH-1015 Lausanne, Switzerland (Professor Emeritus) \\ * corresponding author \\ \{luca.dede, alfio.quarteroni,francesco.regazzoni\}@polimi.it
}

This paper is dedicated to the memory of Edoardo Vesentini.

\begin{abstract}
This paper deals with the mathematical model that describes the function of the human heart. More specifically, it addresses the equations that express the electromechanical process, that is the mechanical deformation (contraction and relaxation) of the heart muscle that is induced by the electrical field that, at every heartbeat, is generated in the sino-atrial node and then propagates all across the cardiac cells. After deriving the equations of the mathematical model from basic physical principles, we proceed to their numerical approximations and discuss issues such as stability, accuracy and computational complexity. We close the paper by illustrating a few numerical results on test problems of potential interest for clinical applications.
\end{abstract}

\section{Introduction}

In the human heart, each heartbeat is triggered by an electrical signal, originating from the sinoatrial node, the heart's natural pacemaker consisting of a group of self-excitable cells and located in the upper part of the right atrium. The signal propagates from one cell to another through the two atria (right and left), and reaches the atrioventricular node, located between the atria and the two ventricles. The atrioventricular node acts as a filter for signal propagation in order to ensure that the contraction of the ventricles begins once the blood has passed from the atria into the same ventricles. The electrical signal then propagates from the atrioventricular node through a fast connecting set of fibers, called the Purkinje fibers, reaching the myocardial cells through the so-called Purkinje muscle junctions. Cardiomyocytes, the cells of the heart muscle, are excitable: when electrically stimulated, the electro-chemical balance of the cell membrane changes, giving rise

(C) Copyright 2021, EMS Press (European Mathematical Society)

This preprint has been published in Atti della Accademia Nazionale dei Lincei, Classe di Scienze Fisiche, Matematiche e Naturali. Rendiconti Lincei - Matematica e Applicazioni 32(2): 233-272 (https://doi.org/10.4171/rlm/ 935) 
to a sequence of biochemical processes that determine a significant variation of the cell potential, precisely a rapid depolarization followed by a repolarization. This phenomenon, known as action potential, is due to the opening and closing of ion channels, that are made of proteins located in the cell membrane. The latter becomes permeable to different ions (calcium, potassium, magnesium) thanks to the transmembrane potential, the voltage difference between the inner and outer part of the cell. The ionic fluxes cause a variation of the transmembrane potential and have a feedback effect on the voltage difference itself. Among the various ionic species involved in the dynamics of the action potential, calcium ions play an important role. Calcium is in fact a trigger for muscle contraction: calcium ions induce a complex chain of reactions by generating an active force inside the cardiomyocytes. Finally, thanks to a transmission process between different space-time scales, the active force at the microscopic scales generates a resulting force at the level of the organ, thus giving rise to deformation, or contraction of the ventricles and atria. The joint effect of active and passive force (i.e. the reaction of the myocardium to mechanical stress thanks to its elastic characteristics), altogether with the coordinated action of the atria and ventricles, governs blood fluid dynamics in the four chambers and dynamics valve.

In this paper we will review the derivation of the mathematical model that governs the electromechanical process occurring in the heart. This model, that is obtained by starting from basic physical principles as well some suitable phenomenological laws, consists in a set of coupled partial differential equations (PDEs) and ordinary differential equations (ODEs). The model variables are suitable to describe the transmembrane electric potential, the concentration of the ionic species at cell level, the active mechanical force that is generated at the cardiomyocytes, and the displacement of the heart muscle.

The complexity of this mathematical model, and the consequent lack of exact solutions in closed form, calls for the construction of suitable numerical approximation strategies. We therefore propose a thorough methodological framework to allow for the numerical construction of an approximate system of nonlinear algebraic equations. We will comment on the stability and convergence properties of the numerical solution and will indicate a possible way to solve this large dimensional system efficiently. The conclusive part of this paper will be devoted to the illustratrion of an example of numerical solution of the electromechanical model of a left ventricle.

The paper is organized as follows. First, we discuss the mathematical models describing the cardiac function. Specifically, in Sec. 2 we address the models describing cardiac electrophysiology. Then, in Sec. 3, we focus on the modeling of active force generation and, in Sec. 4, on cardiac active and passive mechanics. After having reviewed, in Sec. 5, the mathematical models describing blood circulation, we present in Sec. 6 the fully coupled multiphysics model of cardiac electromechanics. We then turn to the numerical approximation of this model, first considering the space discretization of the PDEs (Sec. 7) and then considering the time discretization of the coupled model (Sec. 8). We conclude with Sec. 9, illustrating the numerical solution of the electromechanical model in a left ventricle and commenting on the obtained results.

\section{Modeling cardiac electrophysiology}

The driver of the cardiac function is electrophysiology, resulting from chemical and electrical processes taking place at different spatial scales, from subcellular to the the whole organ scale. A mathematical description of these phenomena is based on the translation in mathematical terms of the principles ruling the chemo-electrical activity of ions species at the finest scale; then, by progressively climbing up the hierarchy of spatial scales, a set of equations describing the tissue-level electrophysiological activity is derived. The multiscale model of cardiac electrophysiology is depicted 


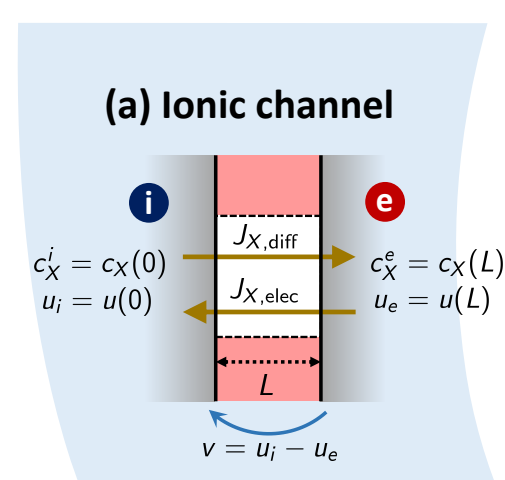

(b) Cell membrane

\section{(e) Organ / ventricle}
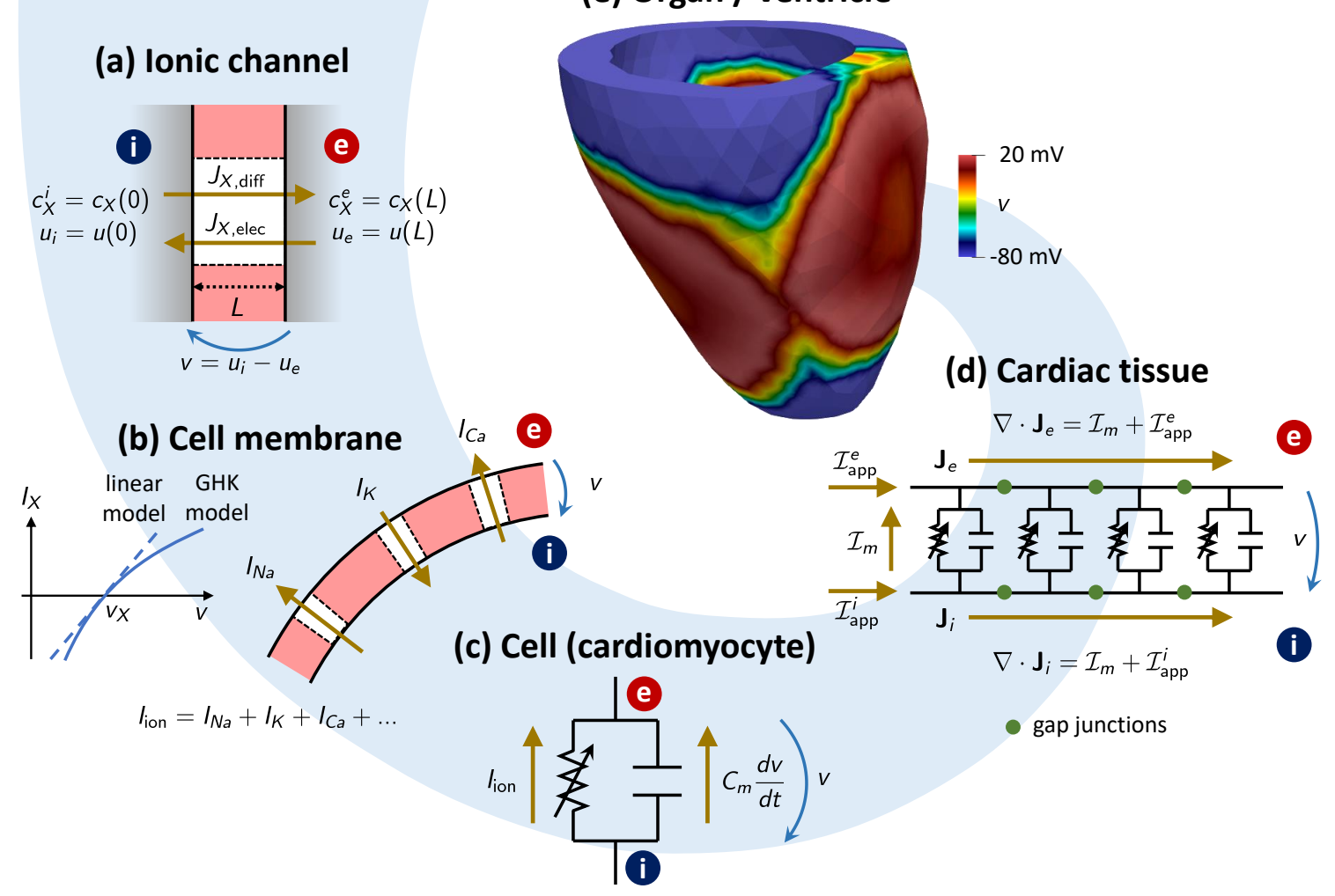

Figure 1: Multiscale model of cardiac electrophysiology. From the finest to the coarsest spatial scale: (a) ionic channels; (b) cell membrane; (c) cardiomyocyte; (d) cardiac tissue; (e) myocardium or cardiac chamber.

in Fig. 1.

\subsection{Transmembrane fluxes of single ionic species}

The exchanges of a ionic species $X$ with valence $z_{X}$ across a permeable membrane is due to ionic fluxes. For simplicity, let us consider a cell membrane with thickness $L$, orthogonal to the $x$ axis, under the assumption that the flux in the tangential direction is negligible compared to the transmembrane one $x$. We indicate such ionic flux as $J_{X}$ for $x \in[0, L]$, which results from the sum of two contributions, namely a diffusive and an electric flux [50] (see Fig. 1a). Diffusion originates a net flux $J_{X \text {,diff }}\left(\mathrm{mol} \mathrm{m}^{-2} \mathrm{~s}^{-1}\right)$ down the concentration gradient, according to the Fick's law $J_{X \text {,diff }}=-D \frac{d c_{X}}{d x}$, where $D$ is the diffusion coefficient $\left(\mathrm{m}^{2} \mathrm{~s}^{-1}\right)$ and $c_{X}$ the ion concentration $\left(\mathrm{mol} \mathrm{m}^{-3}\right)$. On the other hand, the spatial variations of an electric potential $u(\mathrm{~V})$ generate a flux that satisfies the Planck equation $J_{X \text {,elec }}=-c_{X} \operatorname{sign}\left(z_{X}\right) \mu \frac{d u}{d x}$, being $\mu$ the ionic mobility $\left(\mathrm{m}^{2} \mathrm{~V}^{-1} \mathrm{~s}^{-1}\right)$. In his theory on Brownian motion, Einstein linked diffusivity $D$ to $\mu[23]$ as $D=\frac{\mu R T}{\left|z_{X}\right| F}$, where $R$ is the gas constant, $T$ the absolute temperature and $F$ the Faraday constant. It follows that the total ionic flux across 
the membrane returns the Nernst-Plank equation:

$$
J_{X}=J_{X, \text { diff }}+J_{X, \text { elec }}=-D\left(\frac{d c_{X}}{d x}+c_{X} \frac{z_{X} F}{R T} \frac{d u}{d x}\right) .
$$

By denoting with $u_{i}=u(0)$ and $u_{e}=u(L)$ the intracellular andextracellular electrical potentials, respectively, and assuming a constant electric field across the membrane, we get

$$
\frac{d u}{d x}=-\frac{v}{L},
$$

where $v=u_{i}-u_{e}$ is the transmembrane potential. By the continuity equation, we have $\partial_{t} c_{X}+$ $\partial_{x} J_{X}=0$, which entails, at the steady-state, that $J_{X}$ is constant across the cell membrane. It follows from (1) and (2) that

$$
D \frac{d c_{X}}{d x}=\frac{D z_{X} F v}{R T L} c_{X}-J_{X}
$$

whose solution is $c_{X}(x)=\frac{J_{X} R T L}{D z_{X} F v}\left[1-\exp \left(\frac{z_{X} F v}{R T} x\right)\right]+c_{X}(0) \exp \left(\frac{z_{X} F v}{R T} x\right)$.

By defining the electrical current density associated with the ionic flux as $I_{X}:=z_{X} F J_{X}$ $\left(\mathrm{A} \mathrm{cm}^{-2}\right)$, we finally obtain the Goldman-Hodkin-Katz (GHK) current-voltage relation:

$$
I_{X}=\frac{D z_{X}^{2} F^{2}}{L R T} \frac{c_{X}^{i}-c_{X}^{e} \exp \left(-\frac{z_{X} F v}{R T}\right)}{1-\exp \left(-\frac{z_{X} F v}{R T}\right)} v
$$

where $c_{X}^{i}=c_{X}(0)$ and $c_{X}^{e}=c_{X}(L)$ represent the intracellular and extracellular concentrations of the ionic species $X$, respectively.

At thermodynamical equilibrium, the rate of each process is balanced by it reverse, that is $J_{X}=0$. Therefore, the solution of the Nernst-Plank equation (1) entails that, at equilibrium, the transmembrane potential depends on the ratio between the concentration of the ionic species inside and outside the cell membrane. Precisely, the equilibrium potential difference, known as Nernst potential, is $v_{X}=\frac{R T}{z_{X} F} \log \left(\frac{c_{X}^{e}}{c_{X}^{e}}\right)$. The GHK equation is not the unique current-voltage relation used in cardiac electrophysiology models. For example, the following simple voltage-current relationship

$$
I_{X}=g_{X}\left(v-v_{X}\right),
$$

where $g_{X}$ is the membrane conductance, is derived by perturbation analysis from (4) in the longchannel limit (it represents the linearization of GHK). Importantly, both relationships satisfy $I_{X}=0$ when $v=v_{X}$.

\subsection{Cell membrane models}

Equations like (4) and (5) describe the voltage-current relationship of a single ionic species. However, the membrane of cardiomyocytes is permeable to multiple ionic species, as sodium, potassium and calcium. The total electric flux across the cell membrane, as depicted in Fig. 1b, is given by the sum of the electric currents associated with these ionic species, numbered from $k=1$ to $N$ :

$$
I_{\mathrm{ion}}=\sum_{k=1}^{N} I_{k}
$$

where the current of the $k$-th ionic species is modeled either by (4) or (5). 
The cardiomyocytes membrane is selectively permeable to specific ionic species. Equations (4) and (5) describe the voltage-current relationship when all the ionic channels are open. In order to track the opening and closing of these channels, the gating-variables $w_{j} \in[0,1]$, for $j=1, \ldots, M$, are introduced (we have $w_{j}=0$ when all the channels of type $j$ are closed, $w_{j}=1$ when they are all open, $0<w_{j}<1$ when only a fraction of channels are open). Then, considering for instance the linear relationship (5), the net current associated with the $k$-th species is

$$
I_{k}=g_{k}\left(\prod_{j=1}^{M} w_{j}^{p_{j, k}}\right)\left(v-v_{k}\right), \quad \text { for } k=1, \ldots, N,
$$

where $p_{j, k}$ quantifies the influence of the $j$-th channels on the $k$-th ionic species (in particular, we have $p_{j, k}=0$ when the $j$-th channel is not permeable to the $k$-th ion). The dynamics of ionic channels is driven by the transmembrane potential $v$ and according to the following equation:

$$
\frac{d w_{j}}{d t}=\frac{w_{j}^{\infty}(v)-w_{j}}{\tau_{j}}, \quad \text { for } j=1, \ldots, M .
$$

The ionic models described by (6)-(7)-(8) are known as first generation models (e.g. [8, 57]). The most celebrated, the Hodgkin-Huxley model [40], encompasses three ionic currents (sodium current, potassium current and leakage current) and three gating variables:

$$
I_{N a}=g_{N a} w_{1}^{3} w_{2}\left(v-v_{N a}\right), \quad I_{K}=g_{K} w_{3}^{4}\left(v-v_{K}\right), \quad I_{L}=g_{L}\left(v-v_{L}\right) .
$$

Second generation models include an additional set of variables tracking the dynamics of ion concentrations; see e.g. [56, 87-89]. Reduced (phenomenological) models, which provide a phenomenological description of the action-potential dynamics by describing simplified subcellular processes or disregarding them, have also been proposed; see e.g. [1, 11, 26].

Each of the above mentioned ionic models can be written in the general form

$$
\frac{d \mathbf{z}_{\text {ion }}}{d t}=\boldsymbol{\Phi}_{\text {ion }}\left(v, \mathbf{z}_{\text {ion }}\right), \quad I_{\text {ion }}=\mathscr{I}_{\text {ion }}\left(v, \mathbf{z}_{\text {ion }}\right),
$$

where $\mathbf{z}_{\text {ion }}(t) \in \mathbb{R}^{N_{\text {ion }}}$ is a vector collecting the so-called ionic variables (describing ionic channels, concentrations or simply phenomenological variables) and $\mathscr{I}_{\text {ion }}$ and $\boldsymbol{\Phi}_{\text {ion }}$ are suitably defined functions.

To complete a model describing the electrical activity across the cell membrane, we need an equation relating the dynamics of the transmembrane potential $v$ to the total ionic current $I_{\text {ion. }}$. With this aim, the cell membrane is modeled as a capacitor in parallel with a nonlinear resistor, associated with the ionic currents (Fig. 1c):

$$
C_{m} \frac{d v}{d t}+I_{\mathrm{ion}}=I_{\mathrm{app}}
$$

where $C_{m}$ denotes the membrane capacity and $I_{\text {app }}$ represents an externally applied current. The form of $I_{\text {ion }}$ depends on the specific ionic model at hand.

\subsection{Cardiac electrophysiology}

Equation (11) describes the electrical activity of a single cell. However, in the cardiac tissue, the nearly three billion cells are not electrically isolated. Indeed, they are connected by the so-called 
gap junctions, which link the intracellular and extracellular spaces of adjacent cells (see Fig. 1d). Let $\Omega_{0} \subset \mathbb{R}^{3}$ be an open connected set, denoting the region of space occupied by the cardiac tissue. We denote by $\boldsymbol{J}_{e}(\mathbf{X}, t)$ and by $\boldsymbol{J}_{i}(\mathbf{X}, t)$ the local average current densities per unit area in the intracellular and extracellular spaces, respectively. Equation (11), that provides the balance of electric current per unit area across the cell membrane, can be upscaled at the macroscale by introducing the factor $\chi_{m}$, denoting the area-to-volume ratio (i.e. the amount of membrane area per volume of tissue). In fact, the transmembrane current per unit volume can be computed as

$$
\mathcal{I}_{m}=\mathcal{I}_{m}\left(C_{m} \frac{\partial v}{\partial t}+I_{\text {ion }}\right)
$$

Note that we use here partial derivatives, as the variable $v(\mathbf{X}, t)$ is now function of both space and time. Moreover, the conservation of charge entails that the divergence of both $\boldsymbol{J}_{e}$ and $\boldsymbol{J}_{i}$ are balanced by the current $\mathcal{I}_{m}$ and - possibly - by an externally applied current:

$$
\nabla \cdot \boldsymbol{J}_{e}=\mathcal{I}_{m}+\mathcal{I}_{\text {app }}^{e}, \quad \nabla \cdot \boldsymbol{J}_{i}=-\mathcal{I}_{m}+\mathcal{I}_{\text {app }}^{i} .
$$

Finally, we need to relate the electric currents $\boldsymbol{J}_{e}$ and $\boldsymbol{J}_{i}$ to the transmembrane potential $v=u_{i}-u_{e}$. With this aim, the cardiac tissue is typically modeled as an anisotropic medium, whose preferential directions are defined by the arrangement of cardiac cells into fibers and sheets. At each point $\mathbf{X} \in \Omega_{0}$ we define a local frame of reference $\left(\mathbf{f}_{0}, \mathbf{s}_{0}, \mathbf{n}_{0}\right)$, consisting of three orthogonal vectors representing respectively the fibers direction, the sheets normal and a third direction, normal to the others. Denoting by $\sigma_{\alpha}^{f}, \sigma_{\alpha}^{s}$ and $\sigma_{\alpha}^{n}$ (for $\alpha \in\{e, i\}$ ) the extracellular and intracellular conductivity in the directions $\mathbf{f}_{0}, \mathbf{s}_{0}$ and $\mathbf{n}_{0}$, respectively, the conductivity tensors in the extracellular and intracellular media are defined as

$$
\mathbf{D}_{\alpha}=\sigma_{\alpha}^{f} \mathbf{f}_{0} \otimes \mathbf{f}_{0}+\sigma_{\alpha}^{s} \mathbf{s}_{0} \otimes \mathbf{s}_{0}+\sigma_{\alpha}^{n} \mathbf{n}_{0} \otimes \mathbf{n}_{0} \quad \text { for } \alpha \in\{e, i\} .
$$

Then, the currents in the extracellular and intracellular spaces read

$$
\boldsymbol{J}_{e}=-\mathbf{D}_{e} \nabla u_{e}, \quad \boldsymbol{J}_{i}=-\mathbf{D}_{i} \nabla u_{i} .
$$

By combining (12), (13) and (15), we obtain the so-called Bidomain equation:

$$
\begin{array}{rlr}
-\chi_{m} C_{m} \frac{\partial v}{\partial t}-\nabla \cdot\left(\mathbf{D}_{e} \nabla u_{e}\right)-\chi_{m} I_{\text {ion }} & =\mathcal{I}_{\text {app }}^{e} & \text { in } \Omega_{0} \times(0, T], \\
\chi_{m} C_{m} \frac{\partial v}{\partial t}-\nabla \cdot\left(\mathbf{D}_{i} \nabla u_{i}\right)+\chi_{m} I_{\text {ion }} & =\mathcal{I}_{\text {app }}^{i} & \text { in } \Omega_{0} \times(0, T],
\end{array}
$$

On the boundary of the domain $\Omega_{0}$, assuming that the muscle is electrically isolated from the surrounding environment, one typically sets no flux boundary conditions $\left(\mathbf{D}_{e} \nabla u_{e}\right) \cdot \mathbf{N}=\left(\mathbf{D}_{i} \nabla u_{i}\right)$. $\mathbf{N}=\mathbf{0}$, where $\mathbf{N}$ denotes the outward unit vector normal to the surface $\partial \Omega_{0}$.

In spite of our heuristic derivation of the Bidomain model, based on the assumption of interpenetrating domains (the intracellular and extracellular spaces coexist at each point of $\Omega_{0}$ ) [33, 81], this model can be rigorously derived by assuming the existence of the extracellular and intracellular spaces as two simply-connected subdomains and by employing a homogenization technique [18].

By assuming $\mathbf{D}_{e}=\gamma \mathbf{D}_{i}$ for come constant $\gamma>0$ and defining the effective conductivity tensor and the effective applied current as $\mathbf{D}=\frac{\gamma}{1+\gamma} \mathbf{D}_{i}$ and $\mathcal{I}_{\text {app }}=\left(\mathcal{I}_{\text {app }}^{e}+\gamma \mathcal{I}_{\text {app }}^{i}\right) /(1+\gamma)$, (16) reduces to the simpler Monodomain equation:

$$
\chi_{m} C_{m} \frac{\partial v}{\partial t}-\nabla \cdot(\mathbf{D} \nabla v)+\chi_{m} I_{\text {ion }}=\mathcal{I}_{\text {app }} \quad \text { in } \Omega_{0} \times(0, T] .
$$


To wrap up, the electrophysiology models reads

$$
\begin{cases}\chi_{m} C_{m} \frac{\partial v}{\partial t}-\nabla \cdot(\mathbf{D} \nabla v)+\chi_{m} \mathscr{I}_{\text {ion }}\left(v, \mathbf{z}_{\text {ion }}\right)=\mathcal{I}_{\text {app }} & \text { in } \Omega_{0} \times(0, T] \\ \frac{\partial \mathbf{z}_{\text {ion }}}{\partial t}=\boldsymbol{\Phi}_{\text {ion }}\left(v, \mathbf{z}_{\text {ion }}\right) & \text { in } \Omega_{0} \times(0, T]\end{cases}
$$

with suitable initial conditions for $v$ and $\mathbf{z}_{\text {ion }}$ and boundary conditions for $v$ and where $\mathscr{I}_{\text {ion }}\left(v, \mathbf{z}_{\text {ion }}\right)$ and $\boldsymbol{\Phi}_{\text {ion }}\left(v, \mathbf{z}_{\text {ion }}\right)$ are assigned by the membrane model (see Sec. 2.2). The Bidomain model is more detailed than the Monodomain model, even if the latter is by far the most used in application. For the sake of space, in this paper we will only consider the Monodomain model. The applied current $\mathcal{I}_{\text {app }}$ is typically prescribed as a function with support restricted to a short time interval (usually a few milliseconds) at the beginning of each heartbeat and to a few spots located close to the surface of the myocardium, representing the end points of the Purkinje fibers. The typical solution of (18) features a traveling wave for the variable $v$, originating from the regions where $\mathcal{I}_{\text {app }}$ is applied. When a point of the domain is reached by the wavefront, $v$ quickly raises (depolarization), then features a plateau and finally returns to its resting position (repolarization); this yields the so called action potential. Due to the quick depolarization, the potential wave is characterized by a very steep front.

Even when it is derived from physical principles, the mathematical meaningfulness of a model is not guaranteed a priori yet. Specifically, one should check that the problem is well posed from a mathematical standpoint, that is a solution exists, it is unique and it depends continuously from data (the importance of the latter assumption is related to the uncertainty that unavoidably affects the measurements of these data). Moreover, the existence of solutions is an essential requirement to address the numerical approximation of a model. As a matter of fact, even if the approximate problem admits solutions, they would be meaningless in absence of an exact solution they can converge to. In this regard, a well-posedness result for the Bidomain model, in case the FitzhughNagumo model [26] is employed to describe the ionic activity, is provided in [30]. Another wellposedness result, making use of a fixed-point argument, is presented in [92].

\section{Modeling active force generation}

As a consequence of the action potential dynamics, in the first stages of each heartbeat the calcium concentration inside cardiomyocytes (denoted by $\left[\mathrm{Ca}^{2+}\right]_{\mathrm{i}}$, where $i$ stands for intracellular) quickly raises by nearly one order of magnitude before returning to its resting concentration in nearly $500 \mathrm{~ms}$. Calcium acts in fact as a cellular messenger by triggering the contraction of cardiomyocytes.

At the microscopic scale, cardiac muscle tissue is organized in sarcomeres, cylinder shaped contractile elements of the size of nearly $2 \mu \mathrm{m}$. Sarcomeres are composed of myofilaments: thin filaments, made of proteins (troponin, tropomyosin and actin) and thick filaments, made of myosin (see Fig. 2). The active force is generated by the interaction between actin and myosin, molecular motors capable of transforming the chemical energy of ATP into mechanical work [48].

\subsection{Modeling calcium-driven regulation}

The microscale generation of active force is driven by the variation of intracellular calcium concentration due to the following mechanisms. When the muscle is relaxed (i.e. at low calcium concentration), tropomyosin occupies the actin binding sites, preventing the interaction of the latter with myosin (tropomyosin is said to be in non-permissive configuration). When a calcium ion binds to troponin, this bound induces a conformational change in tropomyosin towards a permissive configuration, thus exposing the actin binding site, so that myosin can bind and generate force. Hence, 


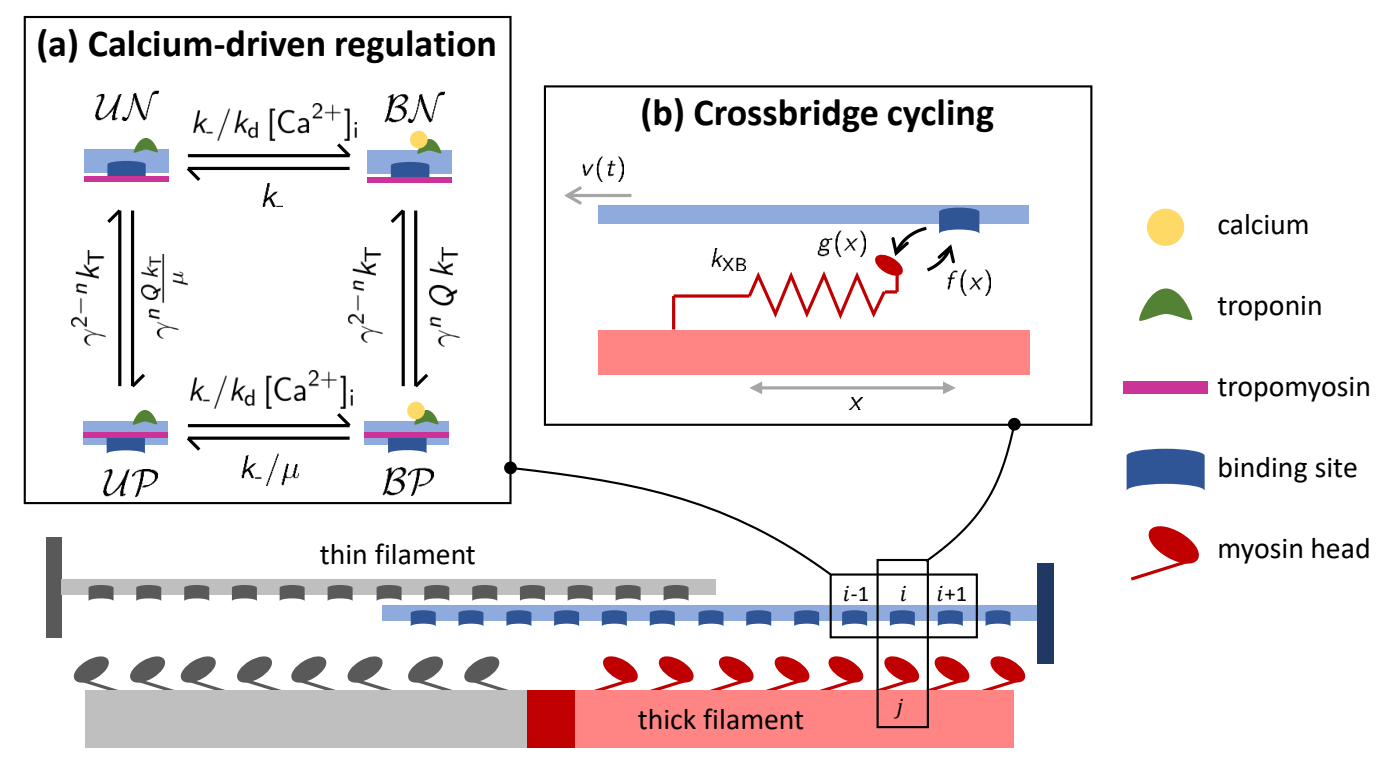

Figure 2: Active force generation model.

by determining the fraction of tropomyosin units in permissive configuration, the concentration of calcium ions inside the cell regulates the amount of generated active force.

In mathematical terms, this process is typically described as a continuous-time Markov Chains (or Markov jump processes, see e.g. [62])). The different configurations of proteins are represented by discrete states, and the transition rates that determine the dynamics of the associated stochastic processes are assigned by the laws of thermodynamics. Let us consider the following simple example. An isolated troponin unit can be in two states: either unbound $(\mathcal{U})$ or bound $(\mathcal{B})$ to a calcium ion. Its dynamics can be described with the following notation

$$
\mathcal{U}+\mathrm{Ca}^{2+} \underset{k_{-}}{\stackrel{k_{+}}{\rightleftharpoons}} \mathcal{B}
$$

where $\rightleftharpoons$ means that two chemical reactions are possible: an unbound troponin unit can bind to a calcium ion to form a bound unit (forward reaction), while a bound unit can turn back to the unbound state releasing a calcium ion (backward reaction). The velocity at which these reactions occurs is described by $k_{+}$and $k_{-}$, respectively called association and dissociation rates. By the law of mass action this dynamics is described in mathematical terms as

$$
\frac{d}{d t}[\mathcal{U}]=k_{-}[\mathcal{B}]-k_{+}[\mathcal{U}]\left[\mathrm{Ca}^{2+}\right]_{\mathrm{i}}, \quad \frac{d}{d t}[\mathcal{B}]=k_{+}[\mathcal{U}]\left[\mathrm{Ca}^{2+}\right]_{\mathrm{i}}-k_{-}[\mathcal{B}]
$$

where $[\mathcal{U}](t)$ (resp. $[\mathcal{B}](t)$ ) denotes the concentration of troponin units in unbound (resp. bound) state at time $t$. At equilibrium, the reverse reaction perfectly balances the forward one and concentrations are constants. At equilibrium we have

$$
\frac{[\mathcal{B}]}{[\mathcal{U}]\left[\mathrm{Ca}^{2+}\right]_{\mathrm{i}}}=\frac{k_{+}}{k_{-}} .
$$

The value of the ratio $k_{+} / k_{-}$can be inferred by thermodynamics arguments. For an ideal dilute solution, the chemical potential (i.e. the Gibbs free energy) of a solute is $G=G^{0}+R T \log \left(c / c_{0}\right)$, 
where $c$ is its concentration, $c_{0}=1 \mathrm{M}$ denotes the standard concentration and $G_{0}$ is the standard free energy at standard concentration $c_{0}$. The change in chemical potential $\Delta G$ associated with the forward reaction of (19) is

$$
\Delta G=G_{\mathcal{B}}-\left(G_{\mathcal{U}}+G_{\mathrm{Ca}^{2+}}\right)=\underbrace{G_{\mathcal{B}}^{0}-\left(G_{\mathcal{U}}^{0}+G_{\mathrm{Ca}^{2+}}^{0}\right)}_{=: \Delta G^{0}}+R T \log \left(\frac{[\mathcal{B}]}{[\mathcal{U}]\left[\mathrm{Ca}^{2+}\right]_{\mathrm{i}}}\right) .
$$

The chemical potential denotes the preference of a state compared to another. At equilibrium, since neither state is preferred, $\Delta G=0$. Therefore, comparing (21) with (22), we obtain the following relationship, that relates the ratio between the transition rates and the difference in standard free energy:

$$
\frac{k_{+}}{k_{-}}=\exp \left(-\frac{\Delta G^{0}}{R T}\right) .
$$

This equation will be later used to describe an important aspect of the dynamics of calcium-driven regulation, that is cooperativity.

Using the formalism of Markov Chains, denoting by $X_{t} \in\{\mathcal{U}, \mathcal{B}\}$ the stochastic process describing the time evolution of the state of a single troponin protein, we can interpret $[\mathcal{U}](t)$ (resp. $[\mathcal{B}](t))$ as the fraction (up to a multiplicative constant) of troponin units in unbound (resp. bound) state at time $t$, that is as the probability $[\mathcal{U}](t)=\mathbb{P}\left[X_{t}=\mathcal{U}\right]$. By summing up the two equations of $(20)$, it follows that, in accordance with the conservation of probability, $[\mathcal{U}](t)+[\mathcal{B}](t)=1$. Hence, $(20)$ reads

$$
\frac{d}{d t}[\mathcal{B}]=k_{+}(1-[\mathcal{B}])\left[\mathrm{Ca}^{2+}\right]_{\mathrm{i}}-k_{-}[\mathcal{B}] .
$$

When $\left[\mathrm{Ca}^{2+}\right]_{\mathrm{i}}$ is constant, the dynamics of $[\mathcal{B}](t)$ asymptotically tends towards an equilibrium state that depends on $\left[\mathrm{Ca}^{2+}\right]_{\mathrm{i}}$, given by the following hyperbolic law (known as Michaelis-Menten equation) [50]:

$$
[\mathcal{B}]_{\text {eq }}=\frac{\left[\mathrm{Ca}^{2+}\right]_{\mathrm{i}}}{\left[\mathrm{Ca}^{2+}\right]_{\mathrm{i}}+\alpha}=\left(1+\frac{\alpha}{\left[\mathrm{Ca}^{2+}\right]_{\mathrm{i}}}\right)^{-1},
$$

where $\alpha=k_{-} / k_{+}$is called half maximal effective concentration, that is - more in general - the concentration of an agent (in this case, calcium ions) producing half of the maximum response.

Mathematical models describing the dynamics of myofilaments are more complex than the twostate reaction (19). Still, the single elementary reactions are modeled using the same mathematical tools. For example, the model of [77] considers, besides the dynamics of troponin, that of tropomyosin, which can be either non-permissive $(\mathcal{N})$ or permissive $(\mathcal{P})$. Thus, the troponintropomyosin complex (known as regulatory unit) can be in one of the four states $\mathcal{U N}, \mathcal{B N}, \mathcal{U P}$ or $\mathcal{B P}$ (see Fig. 2a). The rates associated with the transitions of regulatory units are defined as

$$
\mathcal{U N}+\mathrm{Ca}^{2+} \stackrel{k_{-} / k_{\mathrm{d}}}{\underset{k_{-}}{\rightleftharpoons}} \mathcal{B N} \underset{k_{\mathrm{T}}}{\stackrel{k_{\mathrm{T}} Q}{\rightleftharpoons}} \mathcal{B P} \underset{k_{-} / k_{\mathrm{d}}}{\stackrel{k_{-} / \mu}{\rightleftharpoons}} \mathcal{U P}+\mathrm{Ca}^{2+} \underset{k_{\mathrm{T}} Q / \mu}{\stackrel{k_{\mathrm{T}}}{\rightleftharpoons}} \mathcal{U N}+\mathrm{Ca}^{2+}
$$

where $k_{\mathrm{T}}, k_{\mathrm{d}}, Q$ and $\mu$ are suitable constants. In particular, $\mu \gg 1$ introduces a bias in the dynamics, making the transition $\mathcal{N} \rightarrow \mathcal{P}$ more likely when troponin is bound to calcium (i.e. in state $\mathcal{B}$ ). By denoting, as before, the probability of a state by the symbol $[\cdot]$, the low of mass action entails that the state vector $\mathbf{p}(t)=([\mathcal{U N}](t),[\mathcal{B N}](t),[\mathcal{U P}](t),[\mathcal{B P}](t))^{T}$ evolves according to the linear system

$$
\frac{d \mathbf{p}}{d t}=A \mathbf{p}
$$


The transition matrix

$$
A=\left(\begin{array}{cccc}
-\left(\frac{k_{-}\left[\mathrm{Ca}^{2+}\right]_{\mathrm{i}}}{k_{\mathrm{d}}}+\frac{Q k_{\mathrm{T}}}{\mu}\right) & k_{-} & k_{\mathrm{T}} & 0 \\
\frac{k_{-}\left[\mathrm{Ca}^{2+}\right]_{\mathrm{i}}}{k_{\mathrm{d}}} & -\left(k_{-}+Q k_{\mathrm{T}}\right) & 0 & k_{\mathrm{T}} \\
\frac{Q k_{\mathrm{T}}}{\mu} & 0 & -\left(\frac{k_{-}\left[\mathrm{Ca}^{2+}\right]_{\mathrm{i}}}{k_{\mathrm{d}}}+k_{\mathrm{T}}\right) & \frac{k_{-}}{\mu} \\
0 & Q k_{\mathrm{T}} & \frac{k_{-}\left[\mathrm{Ca}^{2+}\right]_{\mathrm{i}}}{k_{\mathrm{d}}} & -\left(\frac{k_{-}}{k_{\mathrm{d}}}+k_{\mathrm{T}}\right)
\end{array}\right) .
$$

has by construction zero-sum columns, which ensures the conservation of probability (this can be shown by left multiplying (27) by a row vector of ones). System (27) is known as the forward Kolmogorov equation (FKE), or master equation, associated with the CTMC.

An important output of model (27) is the permissivity, that is the fraction of regulatory units in permissive state, defined as $[\mathcal{P}](t)=[\mathcal{U P}](t)+[\mathcal{B P}](t)$. Indeed, by assuming that the actin-myosin interactions occurring for each permissive regulatory units generate a fixed amount of force, the total active force is proportional to the permissivity. The steady-state solution of (27) yields

$$
[\mathcal{P}]_{\mathrm{eq}}=\left(1+\frac{\mu\left(1+\frac{\left[\mathrm{Ca}^{2+}\right]_{\mathrm{i}}}{k_{\mathrm{d}}}\right)}{Q\left(1+\mu \frac{\left[\mathrm{Ca}^{2+}\right]_{\mathrm{i}}}{k_{\mathrm{d}}}\right)}\right)^{-1}
$$

which is well approximated, as $\mu \gg 1$, by the Michaelis-Menten equation

$$
[\mathcal{P}]_{\mathrm{eq}} \simeq[\mathcal{P}]_{\infty}\left(1+\frac{\alpha}{\left[\mathrm{Ca}^{2+}\right]_{\mathrm{i}}}\right)^{-1}, \quad[\mathcal{P}]_{\infty}=\frac{Q}{1+Q}, \quad \alpha=\frac{k_{\mathrm{d}}}{1+Q}
$$

Unfortunately, experimental measurements are in contrast with (29). In fact, the experimentally measured steady-state force-calcium curve does not feature the hyperbolic shape of (29), but rather it shows a good fit with the sigmoidal curve

$$
[\mathcal{P}]_{\mathrm{eq}} \simeq[\mathcal{P}]_{\infty}\left(1+\left(\frac{\alpha}{\left[\mathrm{Ca}^{2+}\right]_{\mathrm{i}}}\right)^{n_{H}}\right)^{-1}
$$

for $n_{H}$ (Hill coefficient) ranging between 4 and 6 [9]. The Hill equation (30) (originally formulated by A. V. Hill to describe binding of oxygen to hemoglobin [38]) models the binding of a ligand to a macromolecule under the hypothesis of cooperative binding. In biochemistry, cooperativity is the phenomenon by which the binding affinity changes when other ligands are already bound to the same macromolecule, thus generating the steep response of (30) near $\alpha$. Cooperativity is of pivotal importance for the function of cardiomyocytes, as it guarantees a rapid and large generation of force in response to small changes in calcium concentration. Without cooperativity, an increase of force from $10 \%$ to $90 \%$ of maximal force would require an 81 -fold increase of $\left[\mathrm{Ca}^{2+}\right]_{\mathrm{i}}$; with an Hill coefficient $n_{H}=5$, to obtain the same force increment, just a factor 2.4 in calcium concentration is enough.

Several hypotheses on the mechanisms leading to the observed cooperative behavior have given rise to mathematical models $[22,24,27,78,80,84]$, but the most likely hypothesis lies in the end-to-end interactions of tropomyosin units [36, 64, 82]. Following [77], let us assume that the configuration with two consecutive tropomyosin units in the same state (either $\mathcal{N}-\mathcal{N}$ or $\mathcal{P}-\mathcal{P}$ ) is energetically more favorable than the case of different state (either $\mathcal{N}-\mathcal{P}$ or $\mathcal{P}-\mathcal{N}$ ). If we denote by $\Delta G_{\mathrm{nn}}$ the difference in free energy between the former and the latter case, by proceeding as above 
(see $(22))$, the transitions $\mathcal{N} \rightleftharpoons \mathcal{P}$ in $(26)$ are redefined as

$$
\mathcal{B N} \frac{\gamma^{n} k_{\mathrm{T}} Q}{\underset{\gamma^{2-n} k_{\mathrm{T}}}{\rightleftharpoons}} \mathcal{B P}, \quad \mathcal{U P} \frac{\gamma^{2-n} k_{\mathrm{T}}}{\underset{\gamma^{n} k_{\mathrm{T}} Q / \mu}{\rightleftharpoons}} \mathcal{U N}
$$

where we have defined $\gamma:=\exp \left(2 \frac{\Delta G_{\mathrm{nn}}}{k_{B} T}\right)$ and $n$ as the number of adjacent units in permissive state (we have $n=0,1,2$ ).

The steady-state solution of the Markov Chain model after the modification (31) (computed in [77] by exploiting the mathematical analogy with the Ising model [17]) is well fitted by the sigmoidal Hill function (30), thus showing a remarkably good agreement with experimental measurements. Moreover, as for the Markov Chain model (26), the time evolution of the probability of each state can be described by the FKE, a linear system of ODEs written in the form (27). However, because of the dependence of the transition rates on the state of the neighboring units, one cannot write an equation for the dynamics of a unit independently of the others, but the vector $\mathbf{p}(t)$ should contain the joint probability of the state of all the units. If we consider a filament with $N=32$ units, this would amount to $4^{N} \simeq 2 \cdot 10^{19}$ variables. Hence, when we face the numerical solution of the FKE, more than $10^{5}$ petabytes would be required just to store the state vector $\mathbf{p}(t)$ in the computer memory, corresponding to more than 30000 times the storage capacity of the largest supercomputer in the world (June 2020). This clearly hinders the possibility of numerically solving the FKE associated with such models.

This situation is not uncommon: a mathematical model is available, although its practical interest is very limited, because of the overwhelming computational cost of its numerical approximation. In these cases, suitable modeling assumptions and/or mathematical tools are employed to derive an approximate, yet computationally feasible, reduced mathematical model. In the case considered in this section, in order to capture the cooperative effects without explicitly tracking the joint probability of the regulatory units of the whole filament, several strategies have been proposed in the literature. Among these, we mention Monte Carlo sampling techniques [94, 95] and the reduction of the number of degrees of freedom obtained by grouping together suitable subsets of the states $[12,53,93]$. Alternatively, in [79] the transition rates of (31) were expressed as nonlinear functions of the calcium concentration, to phenomenologically reproduce the cooperative behavior. In [69], we introduced a physically motivated assumption of conditional independence of the stochastic processes associated with units that are far from each other along the filament, given the state of the intermediate units. With this assumption, the FKE reduces to a nonlinear system of nearly 2000 ODEs, thus allowing to numerically approximate the solution of one heartbeat in just a few seconds of computational time.

\subsection{Modeling the crossbridge cycling}

The long-standing hypothesis that muscle contraction was led by folding of elongated protein filaments was challenged by the discovery that the filaments length remains constant during contraction and that it is instead the mutual sliding between two families of filaments (thin and thick) what makes the muscle contract $[44,47]$. The latter theory, known as sliding filaments theory, was discovered independently by two research teams: on one hand, the British biologist H. Huxley and biophysicist J. Hanson, working at MIT; on the other, the British physiologist A. F. Huxley (Nobel prize winner in 1963 for his work on the action potential) and the German physician R. Niedergerke, working at the University of Cambridge. The two teams decided to publish their work in two consecutive articles in the same issue of Nature [44, 47].

The TOP500 ProJeCt, https://www.top500.org (URL consulted in date January $4^{\text {th }}, 2020$ ). 
The sliding of filaments is caused by the interaction between myosin and actin, which mutually bind by forming the so-called crossbridges. Successively, the head of myosin rotates, pulling the thin filaments and making the muscle fiber contract. The crossbridge is then broken, myosin reattaches at a different position, and the cycle is repeated [9]. As we mentioned in Sec. 3.1, this interaction is only possible when the tropomyosin regulating a given actin binding site is in permissive configuration.

In 1957, A. F. Huxley proposed a celebrated model [46] to describe this subcellular attachmentdetachment process. In this model, myosin is described as a two-state element (either attached or detached), whose transition rates depend on the distance between actin and myosin, denoted by $x$ (see Fig. 2b). We remark that, when the crossbridge is attached, $x$ corresponds to the distortion of the myosin arm, modeled as a linear spring with stiffness $k_{\mathrm{XB}}$. By convention, $x$ is positive when attachment leads to a positive tension. Huxley considered a population of myosin and actin pairs, so large that the probability of finding a pair at distance $x$ is constant within an interval sufficiently close to $x=0$, and he defined $n(x, t)$ the probability that a pair with distance $x$ is attached at time $t$. Under these hypotheses, he derived the following conservation law, for $x \in \mathbb{R}$ and $t \geq 0$ :

$$
\frac{\partial n(x, t)}{\partial t}-v_{\mathrm{hs}} \frac{\partial n(x, t)}{\partial x}=(1-n(x, t)) f(x)-n(x, t) g(x)
$$

where $f(x)$ and $g(x)$ denote the attachment and detachment rates, respectively. In the Huxley model, the probability density $n(x, t)$ is convected by $v_{\mathrm{hs}}=-\frac{1}{2} \frac{d}{d t} S L(t)$, that corresponds to the sliding velocity of the thin filaments relative to the thick filaments $(S L(t)$ denotes the sarcomere length at time $t$ ). The terms at the right-hand side account for the formation and dissolution of crossbridges. Since myosin arms are modeled as linear springs, each attached crossbridge generates an active force of magnitude $k_{\mathrm{XB}} x$. Hence, denoting by $\rho_{\mathrm{AM}}$ the linear density of actin-myosin pairs along a filament and by $\sigma_{\mathrm{hf}}$ the area density of pairs of interacting thin and thick filaments, the tissue-level active tension $T_{\mathrm{a}}(t)$ can be obtained as

$$
T_{\mathrm{a}}(t)=\sigma_{\mathrm{hf}} \rho_{\mathrm{AM}} k_{\mathrm{XB}} \int_{-\infty}^{+\infty} x n(x, t) d x,
$$

by assuming an infinite length of the filaments. To fully define the model, we only need to assign the transition functions $f(x)$ and $g(x)$. In [46], they are phenomenologically set as:

$$
f(x)=f_{1} \frac{x}{h} \chi_{[0, h]}(x), \quad g(x)=g_{2} \chi_{(-\infty, 0]}(x)+g_{1} \frac{x}{h} \chi_{(0,+\infty)}(x),
$$

where $f_{1}, g_{1}$ and $g_{2}$ are positive constants and where $\chi$ is the indicator function. We remark that attachment can occur only in the interval $x \in[0, h]$, that is for positive displacement: such symmetry-breaking feature is what makes the muscle contract. For $x<0$ the detachment rate is very high, in order to prevent the crossbridges to generate force in the opposite direction.

The Huxley model is able of reproducing several experimentally observed features of the phenomenon of active force generation, including the so-called force-velocity relationship, discovered by A. V. Hill, Nobel Prize winner for his work on the heat production and mechanical work in muscles [37]. Specifically, the steady-state solution of the Huxley model for a constant shortening velocity $v_{\text {hs }}$ is such that the active tension is a decreasing function of $v_{\text {hs }}$. However, the Huxley models fails in reproducing some other experimentally observed phenomena, such as the fast-scale response $(\sim 1 \mathrm{~ms})$ to steps either in length or in tension. The reason is that these effects are associated to phenomena (such as the rotation of the myosin heads) that are not explicitly represented in the model. Several generalizations of the Huxley model have been proposed to reproduce also these phenomena, by introducing additional elements to track to rotation of the myosin heads, represented 
either as a discrete variable $[21,45,65,83]$ or as a continuous one $[13,15,16,58,59]$. Nonetheless, we remark that the difference between the classic Huxley models and its generalizations can only be appreciated at tie scales much smaller than those of interest in organ-level simulations [70]. For this reason, these refinements are typically disregarded in multiscale cardiac simulation.

\subsection{Modeling the whole force generation process}

Mathematical models of active force generation in the cardiac tissue include a description of both the calcium-driven activation (see Sec. 3.1) and the crossbridge dynamics (see Sec. 3.2). These two aspects are not independent: only the binding sites associated with a permissive unit are available for crossbridge formation. As a first approximation, this can be taken into account by replacing, in the Huxley model $(32)$, the term $(1-n(x, t))$ with the term $([\mathcal{P}](t)-n(x, t))$, where $[\mathcal{P}](t)$ denotes the overall permissivity (see Sec. 3.1). In [71, 75] a more rigorous coupling between the regulatory units and the crossbridge dynamics is studied: the population of crossbridges is split into two families according to the permissivity state of the associated regulatory unit - and by introducing additional terms accounting for the probability fluxes between the two families. Another model of the whole force generation process is proposed in [51].

A different family is that of phenomenological models $[42,54,55,61,79]$ : these do not derive equations from first principle, rather they are built by fitting the measured data with simple laws, a priori chosen by the modeler. The numerical solution of these models, typically expressed as systems of a few ODEs, feature a lower computational cost than physics-based models, similarly to what happens with cell membrane phenomenological models (Sec. 2.2).

In general terms, force generation models are written in the form

$$
\frac{d \mathbf{z}_{\mathrm{act}}}{d t}=\boldsymbol{\Phi}_{\mathrm{act}}\left(\mathbf{z}_{\mathrm{act}},\left[\mathrm{Ca}^{2+}\right]_{\mathrm{i}}, S L, \frac{d S L}{d t}\right), \quad T_{\mathrm{a}}=q\left(\mathbf{z}_{\mathrm{act}}\right),
$$

where $\mathbf{z}_{\text {act }}(t) \in \mathbb{R}^{N_{\text {act }}}$ denotes a vector collecting the state variables associated with the dynamics of regulatory units and crossbridges.

A family that cannot be strictly written in the form (35) is that of Markov Chain models [43, 94, 95], where the force generation mechanism is described by a unique Markov Chain, including regulatory proteins and crossbridges. Due to the overwhelming dimension of the associated FKE, their numerical approximation is typically based on Monte Carlo techniques.

\section{Modeling cardiac mechanics}

During its normal activity, the heart muscle undergoes large deformations, of the order of few centimeters. To model the myocardium displacement, the strain of the tissue must be related to the internal stress induced by cardiomyocytes' contraction and to the pressure exerted by the blood onto the endocardium. The conceptual framework to describe this phenomenon is continuum mechanics, of which we recall basic notions in the following. For more details, we refer the interested readers to, e.g., $[5,63]$.

\subsection{Kinematics}

We consider an open connected set $\Omega_{0} \subset \mathbb{R}^{d}$, where $d=3$, representing the region of space occupied by an elastic body at rest. We denote $\Omega_{0}$ as the reference (or undeformed) configuration. We then consider a deformation map $\varphi: \Omega_{0} \times[0, T] \rightarrow \mathbb{R}^{d}$, such that $\mathbf{x}=\varphi(\mathbf{X}, t)$ (spatial coordinate) 
represents the position occupied by the point $\mathbf{X} \in \Omega_{0}$ (material coordinate) at time $t$. Furthermore, we define the displacement field $\mathbf{d}(\mathbf{X}):=\varphi(\mathbf{X})-\mathbf{X}$ and the deformation gradient tensor $\mathbf{F}(\mathbf{X}, t)=$ $\nabla \varphi(\mathbf{X}, t)=\mathbf{I}+\nabla \mathbf{d}, \nabla$ being the gradient operator in the reference (material) coordinate and $\mathbf{I}$ being the identity tensor. The time-dependent deformation map is assumed to be smooth enough (typically one assumes twice continuously differentiablility, but weaker regularity can be assumed). Moreover, the deformation map is assumed to be injective and orientation preserving, that is its Jacobian satisfies $J=\operatorname{det} \mathbf{F}>0$.

By denoting by Lin the vector space of the linear transformations from $\mathbb{R}^{d}$ into itself, let us introduce the following subsets:

$$
\operatorname{Lin}^{+}:=\{\mathbf{A} \in \text { Lin s.t. } \operatorname{det} \mathbf{A}>0\}, \quad \operatorname{Orth}^{+}:=\left\{\mathbf{A} \in \operatorname{Lin}^{+} \text {s.t. } \mathbf{A}^{T}=\mathbf{A}^{-1}\right\} .
$$

\subsection{Stress tensors and the momentum equilibrium equation}

By the Cauchy stress theorem [5, 63], based on the action-reaction principle (third Newton law), there exists a second-order symmetric tensor (more precisely, a tensor field), the Cauchy stress tensor $\mathbf{T}$, such that the internal stress across a surface $A$ intersecting the body in the current configuration (possibly belonging to its boundary) equals

$$
\mathbf{t}=\int_{A} \mathbf{T n} d A=\int_{A_{0}} J \mathbf{T F}^{-T} \mathbf{N} d A_{0}=\int_{A_{0}} \mathbf{P N} d A_{0},
$$

where $\mathbf{n}$ denotes the unit vector normal to the surface, being $A_{0}$ the counter-image of $A$ with respect to the deformation map $\varphi(\cdot, t)$ and $\mathbf{P}:=J \mathbf{T F}^{-T}$ the first Piola-Kirchhoff stress tensor (or simply Piola stress tensor). By the Newton second law, the time derivative of the momentum associated with the mass contained in a volume $V_{0} \subset \Omega_{0}$ is balanced by the total force acting on it, given by the sum of internal stresses and of an external distributed load $\mathbf{h}$ (force per unit volume):

$$
\frac{d}{d t} \int_{V_{0}} \rho \frac{\partial \mathbf{d}}{\partial t} d V_{0}=\int_{V_{0}} \mathbf{h} d V_{0}-\int_{\partial V_{0}} \mathbf{P N} d A_{0},
$$

where $\rho$ is the body density. By the divergence theorem and by the arbitrariness of $V_{0}$, the balance of momentum equation for the continuum body $\Omega_{0}$ reads

$$
\rho \frac{\partial^{2} \mathbf{d}}{\partial t^{2}}-\nabla \cdot \mathbf{P}=\mathbf{h} \quad \text { in } \Omega_{0} \times(0, T]
$$

\subsection{Hyperelasticity}

To complete the model, (38) must be supplemented with suitable initial and boundary conditions and with a material constitutive law, that is to say a relationship linking the state of strain of the body with its state of stress. The constitutive law can possibly depend on the rate of strain (e.g. in the case of visco-elastic materials), but for simplicity we consider only the case of elastic materials, thus assuming that the stress tensors can be written in terms of the strain tensor as, i.e., $\mathbf{T}=\mathbf{T}_{\mathbf{F}}(\mathbf{F})$ and $\mathbf{P}=\mathbf{P}_{\mathbf{F}}(\mathbf{F})$. In particular, we focus on hyperelastic materials, characterized by a strain energy density $\mathcal{W}$, such that $\int_{\Omega_{0}} \mathcal{W}(\mathbf{X}) d V_{0}$ gives the total elastic energy stored by the body as a consequence of the deformation, where $\mathcal{W}(\mathbf{X})=\mathcal{W}_{\mathbf{F}}(\mathbf{F}(\mathbf{X}))$ for some $\mathcal{W}_{\mathbf{F}}: \operatorname{Lin}^{+} \rightarrow \mathbb{R} \cup\{+\infty\}$. By definition, hyperelastic materials are such that

$$
\mathbf{P}=\frac{\partial \mathcal{W}}{\partial \mathbf{F}} .
$$


Not every material whose Piola stress tensor can be written as in (39) is physically meaningful. Additionally, some minimal requirements should be satisfied. First, by requiring the material response to be independent of the frame of reference used for its description, we get the property stated in the following definition.

Definition 1. We say that a constitutive law is frame-indifferent if the following equivalent properties hold for any $\mathbf{Q} \in \operatorname{Orth}^{+}, \mathbf{F} \in \operatorname{Lin}^{+}$:

$$
\mathbf{T}_{\mathbf{F}}(\mathbf{Q F})=\mathbf{Q} \mathbf{T}_{\mathbf{F}}(\mathbf{F}) \mathbf{Q}^{T}, \quad \mathbf{P}_{\mathbf{F}}(\mathbf{Q F})=\mathbf{Q} \mathbf{P}_{\mathbf{F}}(\mathbf{F}), \quad \mathcal{W}_{\mathbf{F}}(\mathbf{Q F})=\mathcal{W}_{\mathbf{F}}(\mathbf{F})
$$

Moreover, a reasonable assumption is that an increase in a component of strain should yield an increase in the corresponding component of stress (see e.g. [5]). Such order-preserving (or monotonicity) property can be stated in different forms. Let us consider the following definitions.

Definition 2. We say that the constitutive relationship $\mathbf{P}_{\mathbf{F}}:$ Lin $^{+} \rightarrow$ Lin is strongly order-preserving if

$$
\mathbf{H}: \frac{\partial \mathbf{P}_{\mathbf{F}}}{\partial \mathbf{F}} \mathbf{H}>0
$$

holds for any $\mathbf{F} \in \mathrm{Lin}^{+}$and $\mathbf{H} \in$ Lin. Moreover, we say that it is rank-one order-preserving if (40) holds for any $\mathbf{F} \in \mathrm{Lin}^{+}$and $\mathbf{H}$ of rank one. Finally we say that it is non-strictly strongly orderpreserving or non-strictly rank-one order-preserving, if the corresponding non-strict inequalities $(\geq)$ hold.

We remark that a second order tensor $\mathbf{H}$ is of rank one whenever it can be written as $\mathbf{H}=\mathbf{a} \otimes \mathbf{b}$ for some $\mathbf{a}, \mathbf{b} \in \mathbb{R}^{d}$. For hyperelastic materials with twice differentiable strain energy density, this strong order-preserving property is equivalent to the convexity of the strain energy density itself. However, this requirement is too strong to develop a physically meaningful theory of elasticity. First, it is incompatible with frame-indifference [5]. Moreover, it entails uniqueness of the equilibrium under any given load, even if it may neglect physical admissible solutions, as those featuring buckling. Conversely, the rank-one order-preserving property circumvents the above mentioned drawbacks and, additionally, such materials can be characterized by traveling pressure waves with real velocity $[5]$.

Besides the strong and the rank-one order-preserving properties, intermediate notions have been proposed in the literature, such as that of quasi-convexity and of polyconvexity of the strain energy density, which, together with suitable growth and regularity conditions, allows proving existence of equilibria [6, 60]. The latter results, due to J. Ball, are based on the Direct Method of Calculus of Variation [19]. We notice that quasi-convexity and polyconvexity are stronger conditions than the rank-one order-preserving, but weaker than the strong order-preserving property.

\subsection{Cardiac constitutive relationships}

The passive mechanical response of the heart is significantly anisotropic, due to the presence of fibers. This property is formalized by introducing the material-symmetry group as the class of rotations that do not affect the material response (see [5] for more details). Transversely isotropic materials, for instance, have a symmetric response in the plane orthogonal to a preferential direction, while orthotropic material have three mutually orthogonal preferential directions. Many transversely isotropic or orthotropic constitutive laws have been proposed in the literature to describe the cardiac tissue (see e.g. $[34,35,41,91]$ ), accounting for the different elastic response along the directions $\mathbf{f}_{0}$, $\mathbf{s}_{0}$ and $\mathbf{n}_{0}$. These energies typically feature an exponential dependence on the strain to model the 
large stiffening of the tissue when it is over-stretched. As an example, the material model of [91] is defined by the strain energy density function

$$
\mathcal{W}=\frac{C}{2}\left(e^{Q}-1\right)+\frac{B}{2}(J-1) \log J
$$

where

$$
Q=b_{\mathrm{ff}} E_{\mathrm{ff}}^{2}+b_{\mathrm{ss}} E_{\mathrm{ss}}^{2}+b_{\mathrm{nn}} E_{\mathrm{nn}}^{2}+2 b_{\mathrm{fs}} E_{\mathrm{fs}}^{2}+2 b_{\mathrm{fn}} E_{\mathrm{fn}}^{2}+2 b_{\mathrm{sn}} E_{\mathrm{sn}}^{2},
$$

and where $E_{a b}=\mathbf{E} \mathbf{a}_{0} \cdot \mathbf{b}_{0}$, for $a, b \in\{\mathrm{f}, \mathrm{s}, \mathrm{n}\}$, are the entries of the Green-Saint Venant tensor $\mathbf{E}=\frac{1}{2}\left(\mathbf{F}^{T} \mathbf{F}-\mathbf{I}\right)$ in the $\left(\mathbf{f}_{0}, \mathbf{s}_{0}, \mathbf{n}_{0}\right)$ frame of reference. The constant $B>0$ is called bulk modulus and weighs the volumetric term $\frac{1}{2}(J-1) \log J$ by penalizing those deformations that would lead to a change of the volume occupied by the tissue $(J \neq 1)$. The latter term leads to a quasi-incompressible formulation, as little volume variations are allowed. The strongly incompressible formulation is an alternative, in which the balance of momentum equation (38) is coupled with the constraint $J=1$, and the Piola stress tensor is redefined as $\mathbf{P}=\frac{\partial \mathcal{W}}{\partial \mathbf{F}}-p J \mathbf{F}^{-T}$, thus yielding a saddle-point problem, wherein the pressure $p$ acts as Lagrange multiplier.

\subsection{Cardiac active mechanics}

As a metter of fact, the cardiac tissue is an active material. This means that its internal stress is not uniquely identified by strain, but rather it can be produced by microscopic mechanisms that turn the chemical energy of ATP into mechanical work. The models presented in Sec. 3 describe this process at the microscale and allow to obtain $T_{\mathrm{a}}$, a scalar that represents the magnitude of active force per unit area. This quantity should be related to the macroscopic balance of momentum equation (38), by writing the Piola stress tensor as

$$
\mathbf{P}=\mathbf{P}^{\text {pass }}+\mathbf{P}^{\text {act }}
$$

namely as the sum of a passive term (given by $\mathbf{P}^{\text {pass }}=\frac{\partial \mathcal{W}}{\partial \mathbf{F}}$ ) and an active term $\mathbf{P}^{\text {act }}$, that must be consitutively defined by suitably upscaling the microscopically generated stress. Alternative to the active stress approach (42) is the active strain one, wherein activation is modeled as a prescribed strain $[2,3]$. in this paper, we only consider the active stress approach, which is by far the most used in the literature.

If we suppose that cardiac muscle fibers, aligned along $\mathbf{f}_{0}$, generate a force per unit area of magnitude $T_{\mathrm{a}}$, directed as the fibers direction in the current configuration $\mathbf{f}:=\mathbf{F f}_{0} /\left|\mathbf{F f}_{0}\right|$, we get

$$
\mathbf{P}^{\mathrm{act}}=T_{\mathrm{a}} \frac{\mathbf{F f}_{0} \otimes \mathbf{f}_{0}}{\left|\mathbf{F} \mathbf{f}_{0}\right|}
$$

In an alternative upscaling procedure, proposed in [76], the active Piola stress tensor is derived by means of an energetic analysis of the microscopic force generation processes. Specifically, it is obtained by differentiating with respect to $\mathbf{F}$ an energy density function associated with attached crossbridges, thus leading to

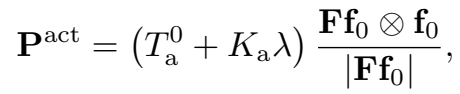

where $\lambda=\left|\mathbf{F f}_{0}\right|-1$ is the fibers stretch, $T_{\mathrm{a}}^{0}$ denotes the active tension for $\lambda=0$ and $K_{\mathrm{a}} \geq 0$ is the active stiffness, i.e. the homogenized overall stiffness of attached crossbridges. Eq. (43) is in the form of (44) as $T_{\mathrm{a}}=T_{\mathrm{a}}^{0}+K_{\mathrm{a}} \lambda$ gives the active tension per unit area in the fibers direction. 
However, in (44) the dependence of the microscopic active force on the tissue stretch is explicit, thus accounting for the fact that crossbridges are modeled as microscopic springs. We remark that, both in (43) and (44), the active part of the Piola stress tensor can be written as

$$
\mathbf{P}^{\text {act }}=\mathbf{P}_{\mathbf{F}}^{\text {act }}(\mathbf{F})=\psi(\lambda) \frac{\mathbf{F f}_{0} \otimes \mathbf{f}_{0}}{1+\lambda},
$$

for a suitable function $\psi(\lambda)$.

We have the following result, whose simple proof is left to the reader.

Proposition 1. Any constitutive law in the form (45) is frame-indifferent.

In virtue of the additive decomposition (42), if the passive constitutive behavior of the material is wither strongly or rank-one order-preserving, then it is sufficient for the total Piola stress tensor to fulfill the same notion that the active part satisfies its non-strict counterparts. Motivated by this observation, we study the order-preserving properties of the active Piola stress tensors belonging to the family of (45). Such properties are fully characterized by the following result.

Proposition 2. Let us consider the Piola stress tensor (45), where $\psi$ is a differentiable function. Then, if

$$
\psi(\lambda) \geq 0, \quad \psi^{\prime}(\lambda) \geq 0 \quad \forall \lambda \in(-1,+\infty),
$$

$\mathbf{P}_{\mathbf{F}}^{\text {act }}$ is non-strictly rank-one order-preserving and is guaranteed to be non-strictly strongly orderpreserving.

Proof. First, we notice that

$$
\mathbf{H}: \frac{\partial \mathbf{P}_{\mathbf{F}}^{\text {act }}}{\partial \mathbf{F}} \mathbf{H}=\frac{\psi(\lambda)}{1+\lambda}\left|\mathbf{H f}_{0}\right|^{2}+\frac{\psi^{\prime}(\lambda)(1+\lambda)-\psi(\lambda)}{(1+\lambda)^{3}}\left(\mathbf{F f}_{0} \cdot \mathbf{H f}_{0}\right)^{2} .
$$

Let us suppose that the non-strict rank-one order-preserving property holds. Let us first consider $\mathbf{H}=\mathbf{a} \otimes \mathbf{b}$, where $\mathbf{b}=\mathbf{f}_{0}$ and $\mathbf{a}$ is a unit vector orthogonal to $\mathbf{F} \mathbf{f}_{0}$. Then, $\left|\mathbf{H f}_{0}\right|^{2}=|\mathbf{a}|^{2}=1$ and $\mathbf{F f}_{0} \cdot \mathbf{H f}_{0}=\mathbf{F f}_{0} \cdot \mathbf{a}=0$ and the sign of the result is that of $\psi(\lambda)$. Thus $\psi(\lambda) \geq 0$ is a necessary condition for the non-strict rank-one order-preserving property. On the other hand, let us take $\mathbf{b}=\mathbf{f}_{0}$ and $\mathbf{a}=\mathbf{F} \mathbf{f}_{0}$. In this case $\mathbf{H f}_{0}=\mathbf{F} \mathbf{f}_{0}$ and the result is $\psi^{\prime}(\lambda)(1+\lambda)^{2}$, whose sign is that of $\psi^{\prime}(\lambda)$. Thus $\psi^{\prime}(\lambda) \geq 0$ is a necessary condition for the non-strict rank-one order-preserving property too.

To show the other implication, we suppose that the inequalities of (46) are satisfied and we consider two cases. First, if $\psi^{\prime}(\lambda)(1+\lambda)-\psi(\lambda) \geq 0$, under the hypothesis $\psi(\lambda) \geq 0$ all the terms are nonnegative, giving the thesis. Instead, if the $\psi^{\prime}(\lambda)(1+\lambda)-\psi(\lambda)<0$, since $\left(\mathbf{F f}_{0} \cdot \mathbf{H f}_{0}\right)^{2} \leq$ $\left|\mathbf{F f}_{0}\right|^{2}\left|\mathbf{H f}_{0}\right|^{2}$, we have

$$
\mathbf{H}: \frac{\partial \mathbf{P}_{\mathbf{F}}^{\text {act }}}{\partial \mathbf{F}} \mathbf{H} \geq \frac{\psi(\lambda)}{1+\lambda}\left|\mathbf{H} \mathbf{f}_{0}\right|^{2}+\frac{\psi^{\prime}(\lambda)(1+\lambda)-\psi(\lambda)}{(1+\lambda)^{3}}\left|\mathbf{F f}_{0}\right|^{2}\left|\mathbf{H f}_{0}\right|^{2}=\psi^{\prime}(\lambda)\left|\mathbf{H f}_{0}\right|^{2} \geq 0,
$$

whence the thesis.

Hence, (46) are also necessary and sufficient conditions for quasi-convexity, that allows to prove existence of equilibria, thanks to the results of J. Ball $[6,19]$. As simple corollaries of Prop. 2, we can derive conditions for which the Piola stress tensors (43) and (44) are compliant with order-preserving properties. In the case of $(43)$, we have $\varphi(\lambda)=T_{\mathrm{a}}$, that is a constant. Therefore, the Piola tensor is strongly order-preserving whenever $T_{\mathrm{a}} \geq 0$. Conversely, the active Piola tensor (44) satisfies the 
order-preserving property just on the set of deformation $\mathbf{F} \in \operatorname{Lin}^{+}$such that $\psi(\lambda)=T_{\mathrm{a}}^{0}+K_{\mathrm{a}} \lambda \geq 0$. Indeed, we have $\psi^{\prime}(\lambda)=K_{\mathrm{a}} \geq 0$ by definition. As noticed above, the quantity $\left(T_{\mathrm{a}}^{0}+K_{\mathrm{a}} \lambda\right)$ corresponds to the generated active force per unit area. Therefore, both (43) and (44) are non-strictly strongly order-preserving as soon as the generated tension is nonnegative. We notice that, for physical meaningfulness, this is always true (unless the tissue is quickly compressed by an external agent, leading to a negative average elongation of attached crossbridges; however, this never happens in a beating heart). In conclusion, both models (43) and (44) are suitable for cardiac modeling, as they satisfy both the frame-indifference and the strain-stress monotonicity requirements.

\section{$5 \quad$ Modeling blood circulation}

The four heart cavities are filled by blood. A further mathematical model should then ideally be used to describe the complex blood dynamics during the different phases of the heartbeat. Blood is a suspension of particles (red blood cells, leukocytes, and platelets) in a fluid bed, the plasma. Its behavior in large arterial vessels as well as in the heart chambers, however, can be assimilated to that of a continuum viscous incompressible flow, that can be modeled by means of the classical NavierStokes equations. The intricacy here is due to the fact that the mathematical domain wherein these equations have to be solved is changing in time according to a dynamics that is itself unknown. Take for instance the case of the left ventricle. The internal cavity represents the mathematical domain for the Navier-Stokes equations. Its boundaries can be regarded as given by the endocardium surface (i.e.the internal membrane of the myocardium), and the cross areas of the two valves that regulate the inflow and outflow of the blood in the left ventricle: the mitral valve, connecting left ventricle and left atrium, and the aortic valve, whose opening allows blood to be ejected into the ascending aorta. The dynamical change of the endocardium during the heartbeat is governed by the deformation of the myocardium, and thus is a result of the electromechanical model that we have derived in the previous section. More precisely, what really governs the blood dynamics is the result of the physical interaction between the two models, the fluid dynamics one and the electromechanical model (actually, it is the mechanical part that matters here). From a mathematical standpoint, this is a fluid-structure interaction (FSI) problem: the fluid is the blood, the structure is represented by the myocardium. Its mathematical conceptualization is as follows: at any time $t$, in one domain (the volume occupied by the fluid at that time) we solve the fluid dynamics equations, in the adjoining domain (the one corresponding to the position occupied by the myocardium at the same time $t$ ) we solve the mechanical problem, while at their interface we need to enforce appropriate coupling conditions. The latter represent the continuity of the velocity fields (this is the kinematic condition) and the equilibrium of dynamical forces (this is the dynamic condition). See [67].

Solving FSI problems numerically is very demanding in terms of computational resources. As a matter of fact, since fluid dynamics equations are typically formulated in an Eulerian frame of reference and solid mechanics equations in a Lagrangian setting, their coupling needs to be accommodated appriopriately. One possible strategy in this respect is to adopt a so-called ALE (arbitrary Lagrangian Eulerian) viewpoint, according to which the Navier-Stokes equations are modified by replacing the temporal derivative of the velocity (the acceleration term in the momentum equations) by a time derivative taken in a reference domain (such as the one corresponding at the fluid at the end diastolic phase) plus a new convective velocity that represents the rate of deformation of the fluid domain itself. This latter term introduces in fact a further unknown in the coupled system. Its determination can be achieved via the solution of a further PDE (e.g. a vector Laplacian) that allows the reconstruction of the domain transformation map in terms of the interface displacement [29]. 
With the aim of reducing the computational burden of FSI coupled problems, several strategies can be envisaged. The perhaps cheapest one consists in barely dropping the fluid equations and using as their surrogate the "force" that the fluid exerts on the solid (the myocardium) at the endocardium interface. This force in fact represents a Neumann boundary condition that must be supplemented to the balance of momentum equation (38). On its end, the blood dynamics in the chambers is tightly related to that of the whole vascular network. This calls for the development of models that are suitable to describe the blood dynamics of the entire circulatory system. Several blood circulation models, featuring different degrees of accuracy, have been proposed. They range from three-dimensional FSI models to be used for large arteries $[67,85,86]$, to zero-dimensional models (whose variables only depend on time, but not on spatial coordinates) [67]. See [28, 68] for a thorough review on the coupling between $3 \mathrm{D}, 1 \mathrm{D}$ and $0 \mathrm{D}$ blood dynamics models. In the latter family of models, also known as lumped parameters models, the circulatory system is split into a finite number of compartments and an average pressure and an average flow rate is associated with each of them. The corresponding equations are derived by the principles of conservation of mass and momentum to be satisfied in every compartment. Lumped parameter models may describe the whole closed-loop, cardio-circulatory system [10,39, 73] models or they may be limited to a subset of the circulatory systems $[14,32]$ In general terms, they are written as systems of ODEs, in the form

$$
\frac{d \mathbf{z}_{\mathrm{circ}}}{d t}=\boldsymbol{\Phi}_{\mathrm{circ}}\left(\mathbf{z}_{\mathrm{circ}}, t\right)
$$

where the vector $\mathbf{z}_{\text {circ }}(t) \in \mathbb{R}^{N_{\text {circ }}}$ collects the state variables (pressure and volumes). The right-hand side may depend on the time variable, to account for the different phases of the cardiac cycle.

An intermediate class of models for vessel circulation consists of $1 \mathrm{D}$ models, in which only the main direction of blood flow is explicitly represented and the equations are mediated along the orthogonal cross section. For the sake of space, 3D and $1 \mathrm{D}$ circulation models will not be further discussed in this paper.

\section{A fully coupled cardiac electromechanics model}

In Secs. 2, 3, 4 and 5 we presented mathematical models describing different physical phenomena occurring, at different spatio-temporal scales along each heartbeat. These phenomena, however, cannot however be understood independently of one another. Indeed they are interconnected by means of a complex and fascinating web of interactions, feedback loops and self-regulation mechanisms aimed at preserving the physiological working regime of the heart and at responding in the most advantageous manner to external stimuli. In Fig. 3 the physical quantities at the basis of these interactions are schematically represented. In mathematical terms, in order to describe the whole cardiac function, the models describing the different physics must be coupled to yield a unique system of PDEs and ODEs.

Let us consider for simplicity a single chamber, namely the left ventricle. Let $\Omega_{0} \subset \mathbb{R}^{3}$ be the region of space occupied by the left ventricle at rest (e.g. at the end of the diastole), whose boundary is split into three mutually disconneted parts, namely $\Gamma_{0}^{\mathrm{endo}}, \Gamma_{0}^{\mathrm{epi}}$ and $\Gamma_{0}^{\mathrm{base}}$, denoting endocardium, epicardium and ventricular base, respectively (see Fig. 3). Then, the fully coupled electromechanical problem consists in finding

$$
\begin{aligned}
& v: \Omega_{0} \times[0, T] \rightarrow \mathbb{R}, \quad \mathbf{d}: \Omega_{0} \times[0, T] \rightarrow \mathbb{R}^{3}, \quad \mathbf{z}_{\text {ion }}: \Omega_{0} \times[0, T] \rightarrow \mathbb{R}^{N_{\text {ion }}}, \\
& \mathbf{z}_{\text {act }}: \Omega_{0} \times[0, T] \rightarrow \mathbb{R}^{N_{\text {act }},} \quad \mathbf{z}_{\text {circ }}:[0, T] \rightarrow \mathbb{R}^{N_{\text {circ }}}, \quad p_{\text {LV }}:[0, T] \rightarrow \mathbb{R}
\end{aligned}
$$


such that, we have:

$$
\begin{cases}\chi_{m} C_{m} \frac{\partial v}{\partial t}-\nabla \cdot\left(J \mathbf{F}^{-1} \mathbf{D F}^{-T} \nabla v\right)+\chi_{m} \mathscr{I}_{\text {ion }}\left(v, \mathbf{z}_{\text {ion }}\right)=\mathcal{I}_{\text {app }} & \text { in } \Omega_{0} \times(0, T] \\ \frac{\partial \mathbf{z}_{\text {ion }}}{\partial t}=\boldsymbol{\Phi}_{\text {ion }}\left(v, \mathbf{z}_{\text {ion }}\right) & \text { in } \Omega_{0} \times(0, T] \\ \left(J \mathbf{F}^{-1} \mathbf{D F}^{-T} \nabla v\right) \cdot \mathbf{N}=\mathbf{0} & \text { on } \partial \Omega_{0} \times(0, T] \\ \frac{\partial \mathbf{z}_{\text {act }}}{\partial t}=\mathbf{\Phi}_{\text {act }}\left(\mathbf{z}_{\mathrm{act}},\left[\mathrm{Ca}^{2+}\right]_{\mathrm{i}}\left(\mathbf{z}_{\text {ion }}\right), S L(\mathbf{d}), \frac{\partial S L(\mathbf{d})}{\partial t}\right) & \text { in } \Omega_{0} \times(0, T] \\ \rho \frac{\partial^{2} \mathbf{d}}{\partial t^{2}}-\nabla \cdot \mathbf{P}=\mathbf{0}, \quad \mathbf{P}=\frac{\partial \mathcal{W}}{\partial \mathbf{F}}+T_{\mathrm{a}}\left(\mathbf{z}_{\mathrm{act}}\right) \frac{\mathbf{F f}_{0} \otimes \mathbf{f}_{0}}{\left|\mathbf{F} \mathbf{f}_{0}\right|} & \text { in } \Omega_{0} \times(0, T] \\ \mathbf{P N}+K_{\mathrm{epi}} \mathbf{d}+C_{\mathrm{epi}} \frac{\partial \mathbf{d}}{\partial t}=\mathbf{0} & \text { on } \Gamma_{0}^{\text {epi }} \times(0, T] \\ \mathbf{P N}=-p_{\mathrm{LV}} J \mathbf{F}^{-T} \mathbf{N} & \text { on } \Gamma_{0}^{\text {endo }} \times(0, T] \\ \mathbf{P N}=p_{\mathrm{LV}}\left|J \mathbf{F}^{-T} \mathbf{N}\right| \mathbf{v}^{\text {base }}(t) & \text { on } \Gamma_{0}^{\text {base }} \times(0, T] \\ \frac{d \mathbf{z}_{\mathrm{circ}}}{d t}=\mathbf{\Phi}_{\mathrm{circ}}\left(\mathbf{z}_{\mathrm{circ}}, p_{\mathrm{LV}}, t\right) & \text { in }(0, T] \\ \mathcal{V}_{\mathrm{LV}}^{3 \mathrm{D}}(\mathbf{d})=\mathcal{V}_{\mathrm{LV}}^{0 \mathrm{D}}\left(\mathbf{z}_{\mathrm{circ}}\right) & \text { in }(0, T]\end{cases}
$$

with initial conditions (in $\left.\Omega_{0} \times\{0\}\right)$

$$
v=v_{0}, \quad \mathbf{d}=\mathbf{d}_{0}, \quad \frac{\partial \mathbf{d}}{\partial t}=\mathbf{0}, \quad \mathbf{z}_{\text {ion }}=\mathbf{z}_{\text {ion }, 0}, \quad \mathbf{z}_{\text {act }}=\mathbf{z}_{\text {act }, 0}, \quad \mathbf{z}_{\text {circ }}=\mathbf{z}_{\text {circ }, 0} .
$$

In (48)-(50), the electrophysiological activity is described through the monodomain equations. Since the displacement of the myocardium (described through the variable $\mathbf{d}$ ) affects the conductivity properties of the tissue (mechano-electrical feedback), Eq. (14) is rephrased in the deformed configuration as

$$
\mathbf{D}=\sigma_{f} \frac{\mathbf{F f}_{0} \otimes \mathbf{F f}_{0}}{\left|\mathbf{F} \mathbf{f}_{0}\right|^{2}}+\sigma_{s} \frac{\mathbf{F s}_{0} \otimes \mathbf{F s}_{0}}{\left|\mathbf{F} \mathbf{s}_{0}\right|^{2}}+\sigma_{n} \frac{\mathbf{F n}_{0} \otimes \mathbf{F n}_{0}}{\left|\mathbf{F} \mathbf{n}_{0}\right|^{2}}
$$

then, the pull-back of $\mathbf{D}$ in the reference configuration $\Omega_{0}$ is replaced into (18).

The calcium concentration $\left[\mathrm{Ca}^{2+}\right]_{\mathrm{i}}$ at each point of the computational domain and at each time $t$ (encoded in the state variable of the ionic model, i.e. $\mathbf{z}_{\text {ion }}$ ) is an input for the force generation model. The latter is solved at each point in $\Omega_{0}$. To provide the missing input to the latter model, namely the sarcomere length and its time derivative, we observe that the elongation of sarcomeres can be obtained as $S L(\mathbf{d})=S L_{0}\left|\mathbf{F f}_{0}\right|$, where $S L_{0}$ is the reference sarcomere length (also known as sarcomere slack length.

The force generation model, on its turn, provides the magnitude of $T_{\mathrm{a}}$, the active force generated at a given point of $\Omega_{0}$ and at a given time $t \in[0, T]$, which is employed in the equation describing the mechanics of the myocardium. The associated boundary conditions model the interaction of the cardiac muscle tissue with the surrounding tissue. Specifically, the boundary condition enforced on $\Gamma_{0}^{\text {endo }}$ models the stress exerted by the blood contained in the ventricle, whose pressure - corresponding to the variable $p_{\mathrm{LV}}$ - is assumed constant in space. Conversely, the boundary condition imposed on $\Gamma_{0}^{\text {epi }}$ encodes an elastic $\left(K_{\text {epi }}\right)$ and viscous $\left(C_{\text {epi }}\right)$ interaction with the pericardium, a thin membrane enclosing the heart [32]. Finally, on the ventricular base we enforce the so-called 


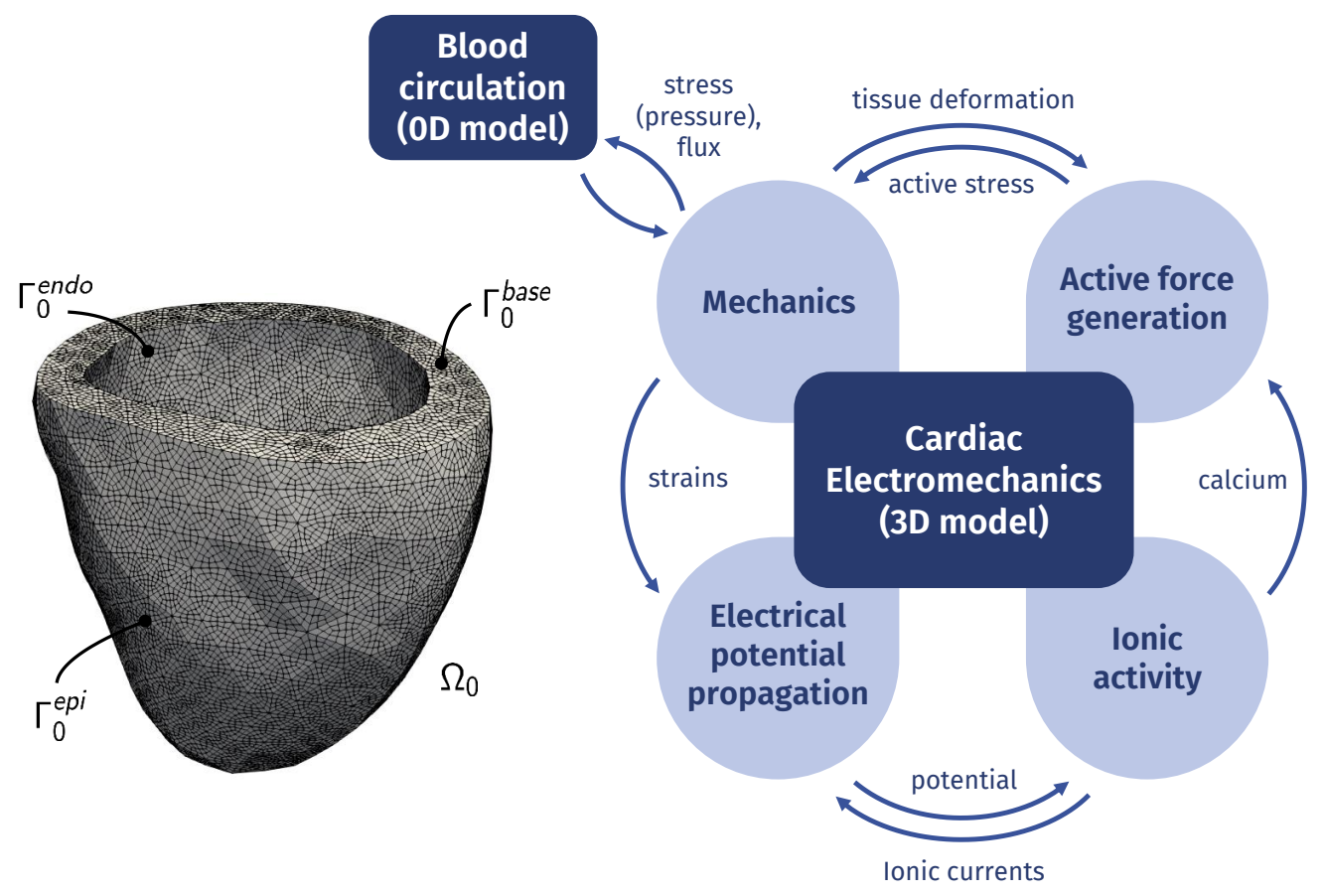

Figure 3: Cardiac electromechanics: computational domain $\Omega_{0}$ and corresponding boundaries $\Gamma_{0}^{\text {epi }}$, $\Gamma_{0}^{\text {endo }}, \Gamma_{0}^{\text {base }}$ (left); core models and cpuling quantities (right).

energy-consistent boundary condition, originally proposed in [72], where we have defined the vector

$$
\mathbf{v}^{\text {base }}(t)=\frac{\int_{\Gamma_{0}^{\text {endo }}} J \mathbf{F}^{-T} \mathbf{N} d \Gamma_{0}}{\int_{\Gamma_{0}^{\text {base }}}\left|J \mathbf{F}^{-T} \mathbf{N}\right| d \Gamma_{0}} .
$$

This condition encodes the effects of the muscle tissue which is not explicitly represented by the computational domain, in an energetically consistent manner.

To couple the three-dimensional model of cardiac electromechanics with the zero-dimensional model of blood circulation, we require that the volume enclosed by the ventricular cavity when the domain $\Omega_{0}$ is moved by the displacement $\mathbf{d}$ - denoted by $\mathcal{V}_{\mathrm{LV}}^{3 \mathrm{D}}(\mathbf{d})$ - is equal to the blood volume contained by the left ventricle within the circulation model - denoted by $\mathcal{V}_{\mathrm{LV}}^{\mathrm{OD}}\left(\mathbf{z}_{\mathrm{circ}}\right)$. The pressure variable $p_{\mathrm{LV}}$ plays the role of Lagrange multiplier, associated with the condition of geometric compatibility $\mathcal{V}_{\mathrm{LV}}^{3 \mathrm{D}}(\mathbf{d})=\mathcal{V}_{\mathrm{LV}}^{0 \mathrm{D}}\left(\mathbf{z}_{\mathrm{circ}}\right)$.

\section{Finite Element approximation}

Let us introduce a hexaedral mesh $\mathcal{T}_{h}$ (tetrahedral meshes are also very common) over the computational domain $\Omega_{0}$ (the left ventricle), where $h$ stands as the maximum diameter of each mesh element $K \in \mathcal{T}_{h}$. Then, let us introduce the set of tensor-products of polynomials with degree smaller than or equal to $r \geq 1$ over the mesh element $K \in \mathcal{T}_{h}$, say $\widetilde{\mathbb{Q}}_{r}(K)$, which are obtained as images on $K$ of the space $\mathbb{Q}_{r}\left(K_{\text {ref }}\right)$ of functions that are polynomials of degree $r$ in each cartesian coordinate over the reference hexaedron $K_{\text {ref. }}$. This allows building the finite dimensional, FE space 
$X_{h}^{r}=\left\{v_{h} \in C^{0}\left(\bar{\Omega}_{0}\right):\left.v_{h}\right|_{K} \in \widetilde{\mathbb{Q}}_{r}(K) \forall K \in \mathcal{T}_{h}\right\}$. For the sake of simplicity, we consider in this paper the case $r=1$, leading to the so called "linear" FE method, for which degrees of freedom are located at the vertexes of the hexaedra; higher order FE approximations $(r \geq 2)$ are however customary in several applications, including cardiac mechanics and electrophysiology. We indicate with $N_{h}=\operatorname{dim}\left(X_{h}^{1}\right)$ the dimension of the former FE space, which is endowed with a Lagrangian FE basis over $\mathcal{T}_{h}$, say $\left\{\varphi_{i}\right\}_{i=1}^{N_{h}}$.

In view of the FE approximation of the EM model (48)-(50), we indicate with $v_{h}(t) \approx v(t)$ the semidiscrete FE approximation of the transmembrane potential $v(t)$, that is $v_{h}(\mathbf{X}, t)=\sum_{i=1}^{N_{h}} \varphi_{i}(\mathbf{X}) v_{i, h}(t)$, where $\underline{\mathbf{v}}_{h}(t)=\left(v_{1, h}(t), \ldots, v_{N_{h}, h}(t)\right)^{T} \in \mathbb{R}^{N_{h}}$ for all $t \in[0, T]$. Similarly, for the displacement variable, we have $\mathbf{d}_{h}(t) \approx \mathbf{d}(t)$, where for this vector field, we need to introduce the vector-valued Lagrangian basis $\left\{\boldsymbol{\varphi}_{i}\right\}_{i=1}^{N_{h}}$ of the FE space $\left[X_{h}^{1}\right]^{3}$. Correspondingly, we express $\mathbf{d}_{h}(t)$ through the vector of time dependent coefficients $\underline{\mathbf{d}}_{h}(t)=\left(\mathbf{d}_{1, h}(t), \ldots, \mathbf{d}_{N_{h}, h}(t)\right)^{T} \in \mathbb{R}^{3 N_{h}}$ for all $t \in[0, T]$.

The ionic and active force generation models in (48)-(50) are formally described by sets of ODEs, whose variables $\mathbf{z}_{\text {ion }}$ and $\mathbf{z}_{\text {act }}$ are defined at every point $\mathbf{X} \in \Omega_{0}$. Following the FE approximation of the potential and displacement variables, we can similarly introduce the corresponding FE approximations $\mathbf{z}_{\text {ion }, h}(t) \in\left[X_{h}^{1}\right]^{N_{\text {ion }}}$ and $\mathbf{z}_{\text {act }, h}(t) \in\left[X_{h}^{1}\right]^{N_{\text {act }}}$, with the notation being understood. Thus, the former can be represented, by introducing the vector-valued Lagrangian basis $\left\{\boldsymbol{\varphi}_{i}\right\}_{i=1}^{N_{h}}$ of the FE spaces $\left[X_{h}^{1}\right]^{N_{\text {ion }}}$ and $\left[X_{h}^{1}\right]^{N_{\text {act }}}$, through vectors of time dependent coefficients $\underline{\mathbf{z}}_{\text {ion }, h}(t)=$ $\left(\mathbf{z}_{\text {ion }, 1, h}(t), \ldots, \mathbf{z}_{\text {ion }, N_{h}, h}(t)\right)^{T} \in \mathbb{R}^{N_{\text {ion }} N_{h}}$ and $\underline{\mathbf{z}}_{\text {act }, h}(t)=\left(\mathbf{z}_{\text {act }, 1, h}(t), \ldots, \mathbf{z}_{\text {act }, N_{h}, h}(t)\right)^{T} \in \mathbb{R}^{N_{\text {act }} N_{h}}$, respectively.

We have now all the notions in place to formulate the Galerkin FE method, which is grounded on the weak formulation of the problem (48)-(50). The fully coupled semidiscrete EM problem consists in finding for every $t \in[0, T]$

$$
\begin{aligned}
& v_{h}(t) \in X_{h}^{1}, \quad \mathbf{d}_{h}(t) \in\left[X_{h}^{1}\right]^{3}, \quad \mathbf{z}_{\text {ion }, h}(t) \in\left[X_{h}^{1}\right]^{N_{\text {ion }}}, \\
& \mathbf{z}_{\text {act }, h}(t) \in\left[X_{h}^{1}\right]^{N_{\text {act }}}, \quad \mathbf{z}_{\text {circ }, h}(t) \in \mathbb{R}^{N_{\text {circ }}}, \quad p_{\mathrm{LV}, h}(t) \in \mathbb{R}
\end{aligned}
$$

such that, we have, in $(0, T]$

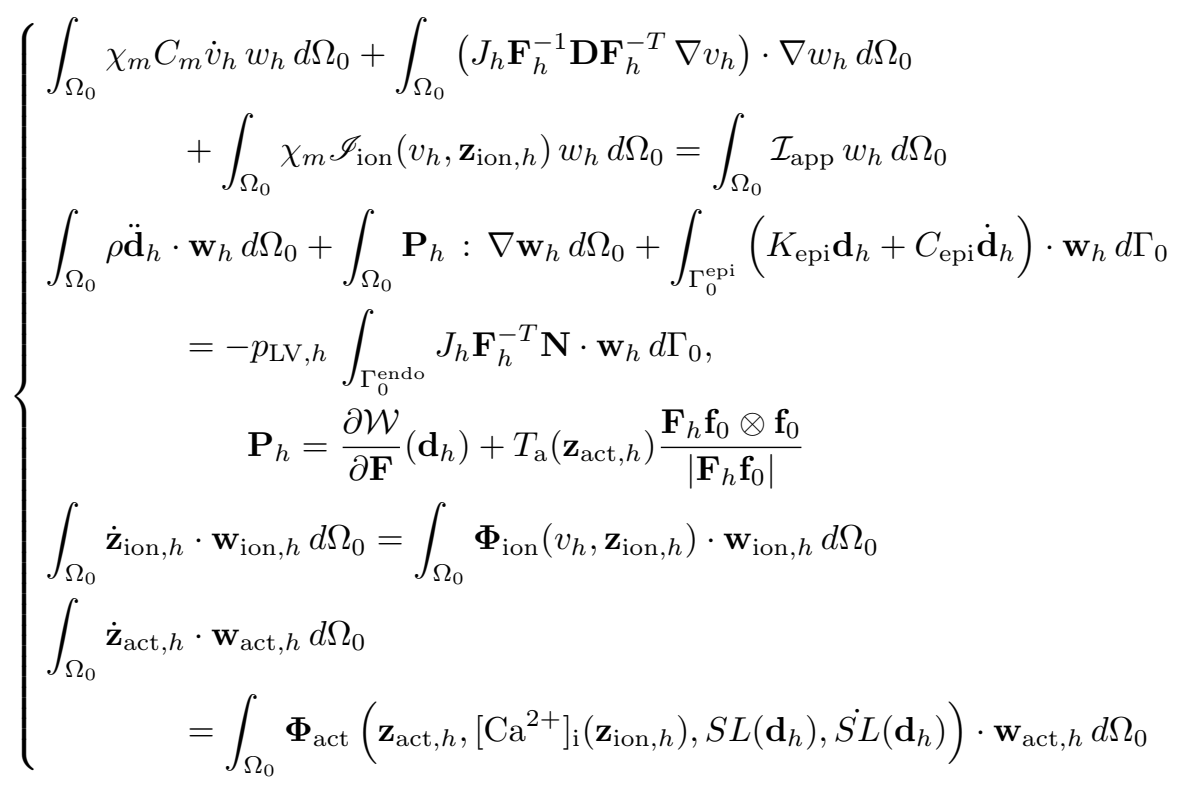


for all the test functions $w_{h} \in X_{h}^{1}, \mathbf{w}_{h} \in\left[X_{h}^{1}\right]^{3}, \mathbf{w}_{\mathrm{ion}, h} \in\left[X_{h}^{1}\right]^{N_{\mathrm{ion}}}$ and $\mathbf{w}_{\text {act }, h} \in\left[X_{h}^{1}\right]^{N_{\text {act }}}$, and coupled with the following equations in $(0, T]$

$$
\dot{\mathbf{z}}_{\mathrm{circ}, h}=\boldsymbol{\Phi}_{\mathrm{circ}}\left(\mathbf{z}_{\mathrm{circ}, h}, p_{\mathrm{LV}, h}, t\right), \quad \mathcal{V}_{\mathrm{LV}}^{3 \mathrm{D}}\left(\mathbf{d}_{h}\right)=\mathcal{V}_{\mathrm{LV}}^{0 \mathrm{D}}\left(\mathbf{z}_{\mathrm{circ}, h}\right)
$$

with initial conditions (in $\left.\Omega_{0} \times\{0\}\right)$

$$
\begin{aligned}
& v_{h}=v_{0, h}, \quad \mathbf{d}_{h}=\mathbf{d}_{0, h}, \quad \dot{\mathbf{d}}_{h}=\mathbf{0}, \quad \mathbf{z}_{\text {ion }, h}=\mathbf{z}_{\text {ion }, 0, h}, \\
& \mathbf{z}_{\text {act }, h}=\mathbf{z}_{\text {act }, 0, h}, \quad \mathbf{z}_{\text {circ }, h}=\mathbf{z}_{\text {circ }, 0} .
\end{aligned}
$$

The initial data for the potential is $v_{0, h}=\sum_{i=1}^{N_{h}} \varphi_{i}\left(v_{0}, \varphi_{i}\right)_{L^{2}\left(\Omega_{0}\right)}$, with the other ones being defined similarly. We remark that $\mathbf{z}_{\mathrm{circ}, h}$ and $p_{\mathrm{LV}, h}$ do not represent the FE approximations of $\mathbf{z}_{\mathrm{circ}}$ and $p_{\mathrm{LV}}$, respectively; rather, the subscript $h$ indicates that these variables are solutions of the coupled semidiscrete problem that accounts for FE approximations of other variables. Finally, we recall that $\mathbf{F}_{h}=\mathbf{I}+\nabla \mathbf{d}_{h}$ and $J_{h}=\operatorname{det}\left(\mathbf{F}_{h}\right)$ in $(53)$.

The algebraic counterpart of the semidiscrete problem (52)-(55) is a system of ODEs. It is obtained by selecting test functions equal to the basis functions of the finite-dimensional function spaces, e.g., $w_{h}=\varphi_{i}$ and $\mathbf{w}_{i}=\boldsymbol{\varphi}_{i}$, and then by performing integrals in the weak formulation through suitable quadrature formulas. The latter are typically Gauss-Legendre formulas over each mesh element $K \in \mathcal{T}_{h}$; see, e.g., [66]. As the ionic and active force generation models were originally sets of ODEs, their FE approximation and assembly of FE matrices and vectors can be circumvented by directly placing the degrees of freedom at the $N_{h}$ vertexes of the mesh $\mathcal{T}_{h}$ that yields the FE space $X_{h}^{1}$. In this manner, the algebraic version of the fully coupled semidiscrete EM problem (52)-(55) consists in finding for every $t \in[0, T]$

$$
\begin{aligned}
& \underline{\mathbf{v}}_{h}(t) \in \mathbb{R}^{N_{h}}, \quad \underline{\mathbf{d}}_{h}(t) \in \mathbb{R}^{3 N_{h}}, \quad \underline{\mathbf{z}}_{\text {ion }, h}(t) \in \mathbb{R}^{N_{\text {ion }} N_{h}}, \\
& \underline{\mathbf{z}}_{\text {act }, h}(t) \in \mathbb{R}^{N_{\text {act }} N_{h}}, \quad \mathbf{z}_{\text {circ }, h}(t) \in \mathbb{R}^{N_{\text {circ }}}, \quad p_{\mathrm{LV}, h}(t) \in \mathbb{R}
\end{aligned}
$$

such that, we have, in $(0, T]$

$$
\left\{\begin{array}{l}
\mathcal{M} \underline{\dot{\mathbf{v}}}_{h}+\mathcal{K}\left(\underline{\mathbf{d}}_{h}\right) \underline{\mathbf{v}}_{h}+\underline{\mathbf{I}}_{\mathrm{ion}}\left(\underline{\mathbf{v}}_{h}, \underline{\mathbf{z}}_{\mathrm{ion}, h}\right)=\underline{\mathbf{I}}_{\mathrm{app}} \\
\mathcal{M}_{s} \underline{\ddot{\mathbf{d}}}_{h}+\mathcal{F} \underline{\dot{\mathbf{d}}}_{h}+\mathcal{G} \underline{\mathbf{d}}_{h}+\underline{\mathbf{S}}\left(\underline{\mathbf{d}}_{h}, \underline{\mathbf{z}}_{\mathrm{act}, h}\right)=-p_{\mathrm{LV}, h} \underline{\mathbf{p}}\left(\underline{\mathbf{d}}_{h}\right) \\
\dot{\underline{\mathbf{z}}}_{\mathrm{ion}, h}=\underline{\boldsymbol{\Phi}}_{\mathrm{ion}}\left(\underline{\mathbf{v}}_{h}, \underline{\mathbf{z}}_{\mathrm{ion}, h}\right) \\
\dot{\dot{\mathbf{z}}}_{\mathrm{act}, h}=\underline{\mathbf{\Phi}}_{\mathrm{act}}\left(\underline{\mathbf{z}}_{\mathrm{act}, h}, \underline{\mathbf{z}}_{\mathrm{ion}, h}, \underline{\mathbf{d}}_{h}, \underline{\dot{\mathbf{d}}}_{h}\right) \\
\dot{\mathbf{z}}_{\mathrm{circ}, h}=\boldsymbol{\Phi}_{\mathrm{circ}}\left(\mathbf{z}_{\mathrm{circ}, h}, p_{\mathrm{LV}, h}, t\right) \\
\mathcal{V}_{\mathrm{LV}}^{3 \mathrm{D}}\left(\underline{\mathbf{d}}_{h}\right)=\mathcal{V}_{\mathrm{LV}}^{0 \mathrm{D}}\left(\mathbf{z}_{\mathrm{circ}, h}\right)
\end{array}\right.
$$

with the corresponding initial conditions (in $\Omega_{0} \times\{0\}$ ). By referring to Eq. (57), we limit to define, e.g.,

$$
\begin{array}{r}
(\mathcal{M})_{i, j}=\int_{\Omega_{0}} \varphi_{j} \varphi_{i} d \Omega_{0}, \quad\left(\mathcal{K}\left(\underline{\mathbf{d}}_{h}\right)\right)_{i, j}=\int_{\Omega_{0}}\left(J_{h} \mathbf{F}_{h}^{-1} \mathbf{D} \mathbf{F}_{h}^{-T} \nabla \varphi_{j}\right) \cdot \nabla \varphi_{i} d \Omega_{0}, \\
\left(\underline{\mathbf{I}}_{\text {ion }}\left(\underline{\mathbf{v}}_{h}, \underline{\mathbf{z}}_{\text {ion }, h}\right)\right)_{i}=\int_{\Omega_{0}} \chi_{m} \mathscr{I}_{\text {ion }}\left(v_{h}, \mathbf{z}_{\text {ion }, h}\right) \varphi_{i} d \Omega_{0}, \quad\left(\underline{\mathbf{I}}_{\text {app }}\right)_{i}=\int_{\Omega_{0}} I_{\text {app }} \varphi_{i} d \Omega_{0},
\end{array}
$$

by leaving the rest of the notation of this algebraic formulation understood. Regarding the nonlinear term $\underline{\mathbf{I}}_{\text {ion }}\left(\underline{\mathbf{v}}_{h}, \underline{\mathbf{z}}_{\text {ion }, h}\right)$, a popular manner to speedup its assembly is using the so-called ionic current 
interpolation (ICI) approach [52]. ICI consists of the approximation

$$
\left(\underline{\mathbf{I}}_{\mathrm{ion}}\left(\underline{\mathbf{v}}_{h}, \underline{\mathbf{z}}_{\mathrm{ion}, h}\right)\right)_{i} \approx \sum_{K \in \mathcal{T}_{h}} \sum_{q=1}^{N_{q}} \sum_{j=1}^{N_{h}} \chi_{m} \mathscr{I}_{\mathrm{ion}}\left(v_{j, h}, \mathbf{z}_{\mathrm{ion}, j, h}\right) \varphi_{j}\left(X_{q}^{K}\right) \varphi_{i}\left(X_{q}^{K}\right) \omega_{q}^{K},
$$

where $\left\{X_{q}^{K}\right\}_{q=1}^{N_{q}}$ are the $N_{q}$ quadrature nodes associated to the mesh element $K \in \mathcal{T}_{h}$ and $\left\{\omega_{q}^{K}\right\}_{k=1}^{N_{q}}$ are the corresponding quadrature weights.

\section{Time discretization}

We consider now the time discretization of the semidiscrete EM problem (56)-(57) in its algebraic formulation. Let us partition for simplicity the time interval $[0, T]$ in $N_{t}$ time instances $t^{n}=n \Delta t$, for $n=0, \ldots, N_{t}$, where the time step size is $\Delta t=\frac{T}{N_{t}}$. To fix the notation, let us consider, e.g., the transmembrane potential $v$, for which we indicate with $\underline{\mathbf{v}}_{h}^{n}=\left(v_{1, h}^{n}, \ldots, v_{N_{h}, h}^{n}\right)^{T} \in \mathbb{R}^{N_{h}}$ the approximation of $\underline{\mathbf{v}}_{h}\left(t^{n}\right)$ for all $n=0, \ldots, N_{t}$; in this manner, the combined FE and time discretizations applied to $v$ will yield the approximation $v\left(t^{n}\right) \approx \sum_{i=1}^{N_{h}} \varphi_{i} v_{i, h}^{n}$. Similar notations hold for the other variables.

We consider first the full discretization, both in space and time, of the EM problem (48)-(50), by using first order accurate backward differentiation formulas (BDF) - a family of multistep methods for the approximation of ODEs (see [66]) - by starting from the semidiscrete fully coupled EM problem (56)-(57). This yields a fully coupled EM problem in the so called monolithic formulation, which consists in finding, for every $n=0, \ldots, N_{t}-1$

$$
\begin{aligned}
& \underline{\mathbf{v}}_{h}^{n+1} \in \mathbb{R}^{N_{h}}, \quad \underline{\mathbf{d}}_{h}^{n+1} \in \mathbb{R}^{3 N_{h}}, \quad \underline{\mathbf{z}}_{\text {ion }, h}^{n+1} \in \mathbb{R}^{N_{\text {ion }} N_{h}}, \\
& \underline{\mathbf{z}}_{\text {act }, h}^{n+1} \in \mathbb{R}^{N_{\text {act }} N_{h}}, \quad \mathbf{z}_{\text {circ }, h}^{n+1} \in \mathbb{R}^{N_{\text {circ }}}, \quad p_{\mathrm{LV}, h}^{n+1} \in \mathbb{R}
\end{aligned}
$$

such that, we have

$$
\left\{\begin{array}{l}
\left(\frac{1}{\Delta t} \mathcal{M}+\mathcal{K}\left(\underline{\mathbf{d}}_{h}^{n+1}\right)\right) \underline{\mathbf{v}}_{h}^{n+1}+\underline{\mathbf{I}}_{\mathrm{ion}}\left(\underline{\mathbf{v}}_{h}^{n+1}, \underline{\mathbf{z}}_{\mathrm{ion}, h}^{n+1}\right)=\frac{1}{\Delta t} \mathcal{M} \underline{\mathbf{v}}_{h}^{n}+\underline{\mathbf{I}}_{\mathrm{app}}^{n+1} \\
\left(\frac{1}{\Delta t^{2}} \mathcal{M}_{s}+\frac{1}{\Delta t} \mathcal{F}+\mathcal{G}\right) \underline{\mathbf{d}}_{h}^{n+1}+\underline{\mathbf{S}}\left(\underline{\mathbf{d}}_{h}^{n+1}, \underline{\mathbf{z}}_{\mathrm{act}, h}^{n+1}\right)+p_{\mathrm{LV}, h}^{n+1} \underline{\mathbf{p}}\left(\underline{\mathbf{d}}_{h}^{n+1}\right) \\
=\left(\frac{2}{\Delta t^{2}} \mathcal{M}_{s}+\frac{1}{\Delta t} \mathcal{F}\right) \underline{\mathbf{d}}_{h}^{n}-\frac{1}{\Delta t^{2}} \mathcal{M}_{s} \underline{\mathbf{d}}_{h}^{n-1} \\
\underline{\mathbf{z}}_{\mathrm{ion}, h}^{n+1}-\Delta t \underline{\mathbf{\Phi}}_{\mathrm{ion}}\left(\underline{\mathbf{v}}_{h}^{n+1}, \underline{\mathbf{z}}_{\mathrm{ion}, h}^{n+1}\right)=\underline{\mathbf{z}}_{\mathrm{ion}, h}^{n} \\
\underline{\mathbf{z}}_{\mathrm{act}, h}^{n+1}-\Delta t \underline{\mathbf{\Phi}}_{\mathrm{act}}\left(\underline{\mathbf{z}}_{\mathrm{act}, h}^{n+1}, \underline{\mathbf{z}}_{\mathrm{ion}, h}^{n+1}, \underline{\mathbf{d}}_{h}^{n+1}, \frac{\underline{\mathbf{d}}_{h}^{n+1}-\underline{\mathbf{d}}_{h}^{n}}{\Delta t}\right)=\underline{\mathbf{z}}_{\mathrm{act}, h}^{n} \\
\mathbf{z}_{\mathrm{circ}, h}^{n+1}-\Delta t \boldsymbol{\Phi}_{\mathrm{circ}}\left(\mathbf{z}_{\mathrm{circ}, h}^{n+1}, p_{\mathrm{LV}, h}^{n+1}, t^{n+1}\right)=\mathbf{z}_{\mathrm{circ}, h}^{n} \\
\mathcal{V}_{\mathrm{LV}}^{3 \mathrm{D}}\left(\underline{\mathbf{d}}_{h}^{n+1}\right)=\mathcal{V}_{\mathrm{LV}}^{0 \mathrm{D}}\left(\mathbf{z}_{\mathrm{circ}, h}^{n+1}\right)
\end{array}\right.
$$

with the corresponding initial conditions. We remark that the time discretization schemes used for the former fully discrete EM formulation, written within the monolithic approach, yields a system of nonlinear algebraic equations to be solved at each time instance $t^{n}$; see, e.g., [32]. As anticipated, this calls for repeatedly using ad hoc methods like the Newton method ([66]), which at each iteration calls 
for assembling Jacobian matrices, other than for solving gigantic, ill-conditioned linear systems. The computational burden associated with (58)-(59) calls for using the so called partitioned or segregated approaches; see, e.g., [20, 31].

We illustrate here an example of a partitioned approach applied to the cardiac EM model (48)(50) and stemming from the semidiscrete problem (56)-(57). The algorithm hereby proposed is inspired by [74] and consists in sequentially solving the following algebraic problems, for every $n=0, \ldots, N_{t}-1$, as follows.

- Ionic model. Solve the algebraic problem for the ionic model by means of the implicit Euler method (equivalent to a first order accurate BDF method) as

$$
\underline{\mathbf{z}}_{\mathrm{ion}, h}^{n+1}-\Delta t \underline{\mathbf{\Phi}}_{\mathrm{ion}}\left(\underline{\mathbf{v}}_{h}^{n}, \underline{\mathbf{z}}_{\mathrm{ion}, h}^{n+1}\right)=\underline{\mathbf{z}}_{\mathrm{ion}, h}^{n},
$$

where $\underline{\mathbf{v}}_{h}^{n}$ is given; the former non linear problem is solved by means of the Newton method.

- Monodomain equation. Solve the problem for the transmembrane potential by means of a semi-implicit scheme associated to the first order accurate BDF method as

$$
\begin{aligned}
\left(\frac{1}{\Delta t} \mathcal{M}\right. & \left.+\mathcal{K}\left(\underline{\mathbf{d}}_{h}^{n}\right)+\frac{\partial}{\partial \mathbf{v}} \underline{\mathbf{I}}_{\text {ion }}\left(\underline{\mathbf{v}}_{h}^{n}, \underline{\mathbf{z}}_{\mathrm{ion}, h}^{n+1}\right)\right) \underline{\mathbf{v}}_{h}^{n+1} \\
& =\left(\frac{1}{\Delta t} \mathcal{M}+\frac{\partial}{\partial \mathbf{v}} \underline{\mathbf{I}}_{\text {ion }}\left(\underline{\mathbf{v}}_{h}^{n}, \underline{\mathbf{z}}_{\mathrm{ion}, h}^{n+1}\right)\right) \underline{\mathbf{v}}_{h}^{n}-\underline{\mathbf{I}}_{\mathrm{ion}}\left(\underline{\mathbf{v}}_{h}^{n}, \underline{\mathbf{z}}_{\mathrm{ion}, h}^{n+1}\right)+\underline{\mathbf{I}}_{\mathrm{app}}^{n+1},
\end{aligned}
$$

where $\underline{\mathbf{d}}_{h}^{n}$ and $\underline{\mathbf{z}}_{\mathrm{ion}, h}^{n+1}$ are given, and additionally the following first order approximation $\underline{\mathbf{I}}_{\text {ion }}\left(\underline{\mathbf{v}}_{h}^{n+1}, \underline{\mathbf{z}}_{\mathrm{ion}, h}^{n+1}\right) \approx$ $\underline{\mathbf{I}}_{\text {ion }}\left(\underline{\mathbf{v}}_{h}^{n}, \underline{\mathbf{z}}_{\text {ion }, h}^{n+1}\right)+\frac{\partial}{\partial \mathbf{v}} \underline{\mathbf{I}}_{\text {ion }}\left(\underline{\mathbf{v}}_{h}^{n}, \underline{\mathbf{z}}_{\mathrm{ion}, h}^{n+1}\right)\left(\underline{\mathbf{v}}_{h}^{n+1}-\underline{\mathbf{v}}_{h}^{n}\right)$ has been used.

- Active force generation. Solve the algebraic problem for active force generation by means of the explicit Euler method

$$
\underline{\mathbf{z}}_{\mathrm{act}, h}^{n+1}-\Delta t \underline{\mathbf{\Phi}}_{\mathrm{act}}\left(\underline{\mathbf{z}}_{\mathrm{act}, h}^{n}, \underline{\mathbf{z}}_{\mathrm{ion}, h}^{n}, \underline{\mathbf{d}}_{h}^{n}, \frac{\underline{\mathbf{d}}_{h}^{n}-\underline{\mathbf{d}}_{h}^{n-1}}{\Delta t}\right)=\underline{\mathbf{z}}_{\mathrm{act}, h}^{n},
$$

given $\underline{\mathbf{d}}_{h}^{n}, \underline{\mathbf{d}}_{h}^{n-1}$, and $\underline{\mathbf{z}}_{\mathrm{ion}, h}^{n+1}$.

- Circulation model. Solve the algebraic circulation model by means of the fourth order accurate, explicit Runge-Kutta method

$$
\mathbf{z}_{\mathrm{circ}, h}^{n+1}=\mathbf{z}_{\mathrm{circ}, h}^{n}+\frac{\Delta t}{6}\left(\mathbf{K}_{1}+2 \mathbf{K}_{2}+2 \mathbf{K}_{3}+\mathbf{K}_{4}\right)
$$

with

$$
\begin{aligned}
& \mathbf{K}_{1}=\boldsymbol{\Phi}_{\mathrm{circ}}\left(\mathbf{z}_{\mathrm{circ}, h}^{n}, p_{\mathrm{LV}, h}^{n}, t^{n}\right) \\
& \mathbf{K}_{2}=\boldsymbol{\Phi}_{\mathrm{circ}}\left(\mathbf{z}_{\mathrm{circ}, h}^{n}+\frac{\Delta t}{2} \mathbf{K}_{1}, p_{\mathrm{LV}, h}^{n}, t^{n}+\frac{\Delta t}{2}\right) \\
& \mathbf{K}_{3}=\boldsymbol{\Phi}_{\mathrm{circ}}\left(\mathbf{z}_{\mathrm{circ}, h}^{n}+\frac{\Delta t}{2} \mathbf{K}_{2}, p_{\mathrm{LV}, h}^{n}, t^{n}+\frac{\Delta t}{2}\right) \\
& \mathbf{K}_{4}=\boldsymbol{\Phi}_{\mathrm{circ}}\left(\mathbf{z}_{\mathrm{circ}, h}^{n}+\Delta t \mathbf{K}_{3}, p_{\mathrm{LV}, h}^{n}, t^{n+1}\right)
\end{aligned}
$$

given $p_{\mathrm{LV}, h}^{n}$. 


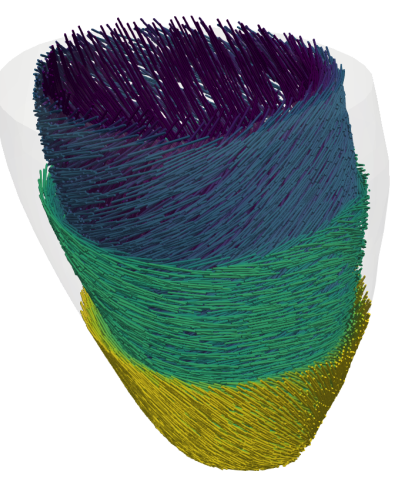

$\mathbf{f}_{0}$

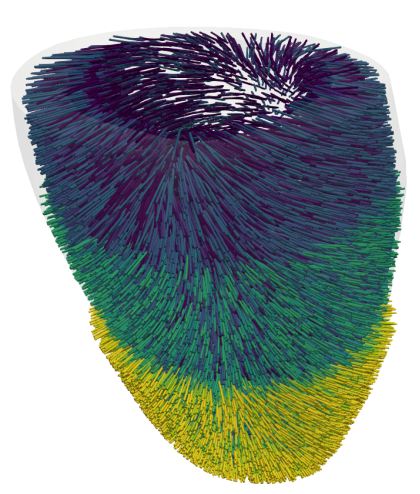

$\mathbf{s}_{0}$

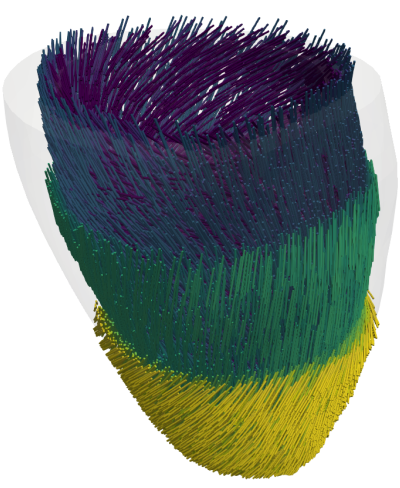

$\mathbf{n}_{0}$

Figure 4: Representation of the fields $\mathbf{f}_{0}, \mathbf{s}_{0}$ and $\mathbf{n}_{0}$, representing the fibers, sheets and cross-fibers directions.

- Tissue mechanics. Solve the following saddle-point, implicit problem for the displacement and pressure variables

$$
\left\{\begin{array}{c}
\left(\frac{1}{\Delta t^{2}} \mathcal{M}_{s}+\frac{1}{\Delta t} \mathcal{F}+\mathcal{G}\right) \underline{\mathbf{d}}_{h}^{n+1}+\underline{\mathbf{S}}\left(\underline{\mathbf{d}}_{h}^{n+1}, \underline{\mathbf{z}}_{\mathrm{act}, h}^{n+1}\right)+p_{\mathrm{LV}, h}^{n+1} \underline{\mathbf{p}}\left(\underline{\mathbf{d}}_{h}^{n+1}\right) \\
=\left(\frac{2}{\Delta t^{2}} \mathcal{M}_{s}+\frac{1}{\Delta t} \mathcal{F}\right) \underline{\mathbf{d}}_{h}^{n}-\frac{1}{\Delta t^{2}} \mathcal{M}_{s} \underline{\mathbf{d}}_{h}^{n-1} \\
\mathcal{V}_{\mathrm{LV}}^{3 \mathrm{D}}\left(\underline{\mathbf{d}}_{h}^{n+1}\right)=\mathcal{V}_{\mathrm{LV}}^{0 \mathrm{D}}\left(\mathbf{z}_{\mathrm{circ}, h}^{n+1}\right)
\end{array}\right.
$$

given $\mathbf{z}_{\mathrm{act}, h}^{n+1}$ and $\mathbf{z}_{\mathrm{circ}, h}^{n+1}$, by using the quasi-Newton strategy outlined in [72].

\section{$9 \quad$ Numerical results}

In this section we show the results of a numerical simulation obtained through the methods presented in Secs. 7-8. Specifically, we here employ a FE discretization in space and a segregated scheme for the numerical coupling of fully discrete core models for which the time discretization is realized by means of BDF schemes. Further details on the numerical schemes employed to obtain the results here presented can be found in [74]. The numerical simulation presented in this section has been car-

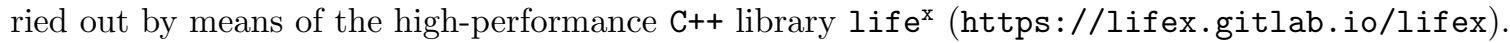
To meet the significant demand of computational resources associated with the numerical approximation of multiscale and multiphysics electromechanics, we rely on the high-performance computing resources available at MOX, Politecnico di Milano (48 Intel Xeon ES-2640 CPUs), exploiting the parallel implementation of the $\operatorname{life} \mathrm{e}^{\mathrm{x}}$ code.

We consider a computational domain $\Omega_{0}$ representing a realistic human left ventricle. The geometry here employed is taken from the Zygote heart model [96], representing an average 21 years old healthy caucasian man. We generate the computational mesh using vmtk (https://www. vmtk . org)[4] and the recently proposed tools for cardiac mesh generation [25]. This computational mesh 


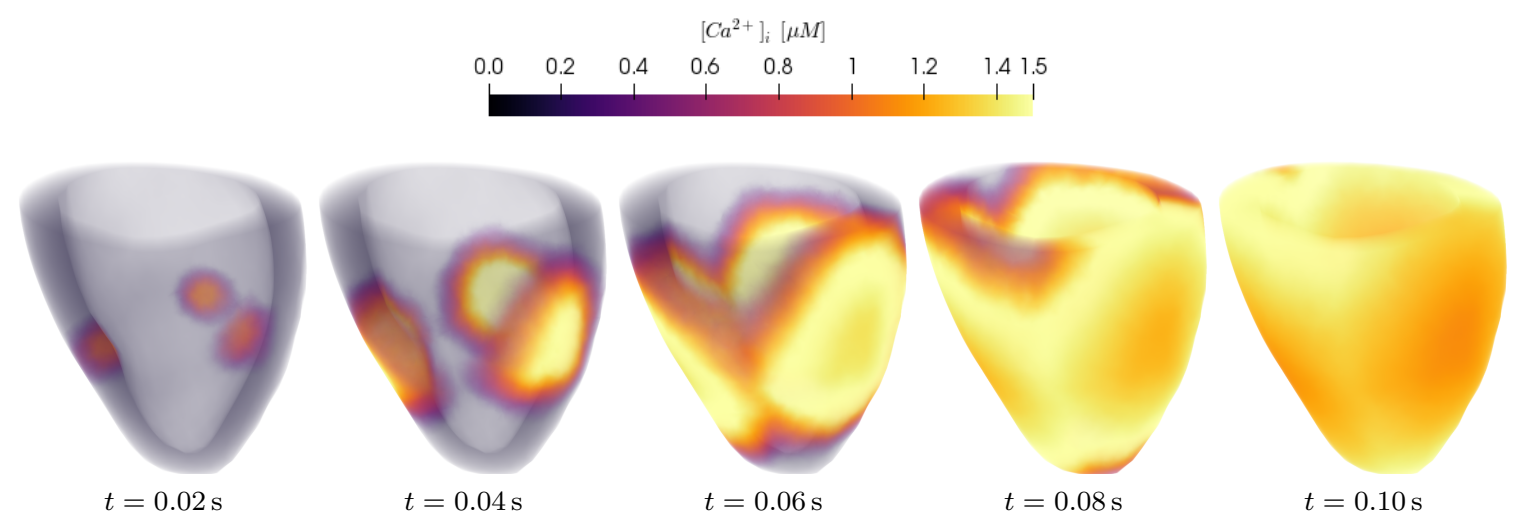

Figure 5: Representation of the intracellular calcium concentration ()$\left.\left[\mathrm{Ca}^{2+}\right]_{\mathrm{i}}\right)$ in the domain $\Omega_{0}$ at different times $t$.

$\mathcal{T}_{h}$, diaplayed in Fig. 3, is made of nearly $350 \cdot 10^{3}$ vertices. For the time discretization we employ a time step size $\Delta t=2 \cdot 10^{-4} \mathrm{~s}$.

The electromechanical model described in this paper requires the definition of the fibers, sheets and cross-fibers directions $\left(\mathbf{f}_{0}, \mathbf{s}_{0}\right.$ and $\left.\mathbf{n}_{0}\right)$. Since these are not explicitly available for the Zygote heart model [96], we we use a so-called rule-based method to generate them following [7]. The generated fields are displayed in Fig. 4.

In Fig. 5 we show the propagation of the variable representing the calcium ions concentration (i.e of the variable $\left[\mathrm{Ca}^{2+}\right]_{\mathrm{i}}$ ) in the computational domain $\Omega_{0}$. The action potential is triggered by means of an electric stimulus $\mathcal{I}_{\text {app }}$ applied in three spherical regions located in the endocardium, to mimic the action of the Purkinje fibers. The calcium dynamics triggers on its turn the microscopic mechanisms of force generation, thus yielding an increase of active tension $T_{\mathrm{a}}$. In Fig. 6 we show the numerical approximation of this latter variable, together with the resulting displacement $\mathbf{d}$ of the tissue.

An advantage of numerical simulations such as the one shown in this section is that they allow to extract information and meaningful indicators of practical use for clinicians. For instance, in Fig. 7 we show the pressure-volume loop (aften abbreviated $P V$ loop), that is a syntetic representation of the left ventricle dynamics in the pressure-volume plane. The PV loop is a fundamental tool in clinical practice, as it allows biomarkers of fundamental importance such as maximum and mean pressures, stroke volume (i.e. identifying the amount of volume pumped by the ventricle per beat) and ejection fraction (i.e. the volumetric fraction of blood ejected from a chamber) to be extracted at a glance $[49,90]$. Moreover, the area enclosed into the pressure-volume curve corresponds to the total mechanical energy generated by the muscle contraction at each heartbeat [49]. In this manner, the outcome of the numerical simulation can be represented in a compact and directly accessible way for the medical doctor.

\section{Acknowledgements}

This project has received funding from the European Research Council (ERC) under the European Union's Horizon 2020 research and innovation programme (grant agreement No 740132, iHEART An Integrated Heart Model for the simulation of the cardiac function, P.I. A. Quarteroni). L. Dedé 


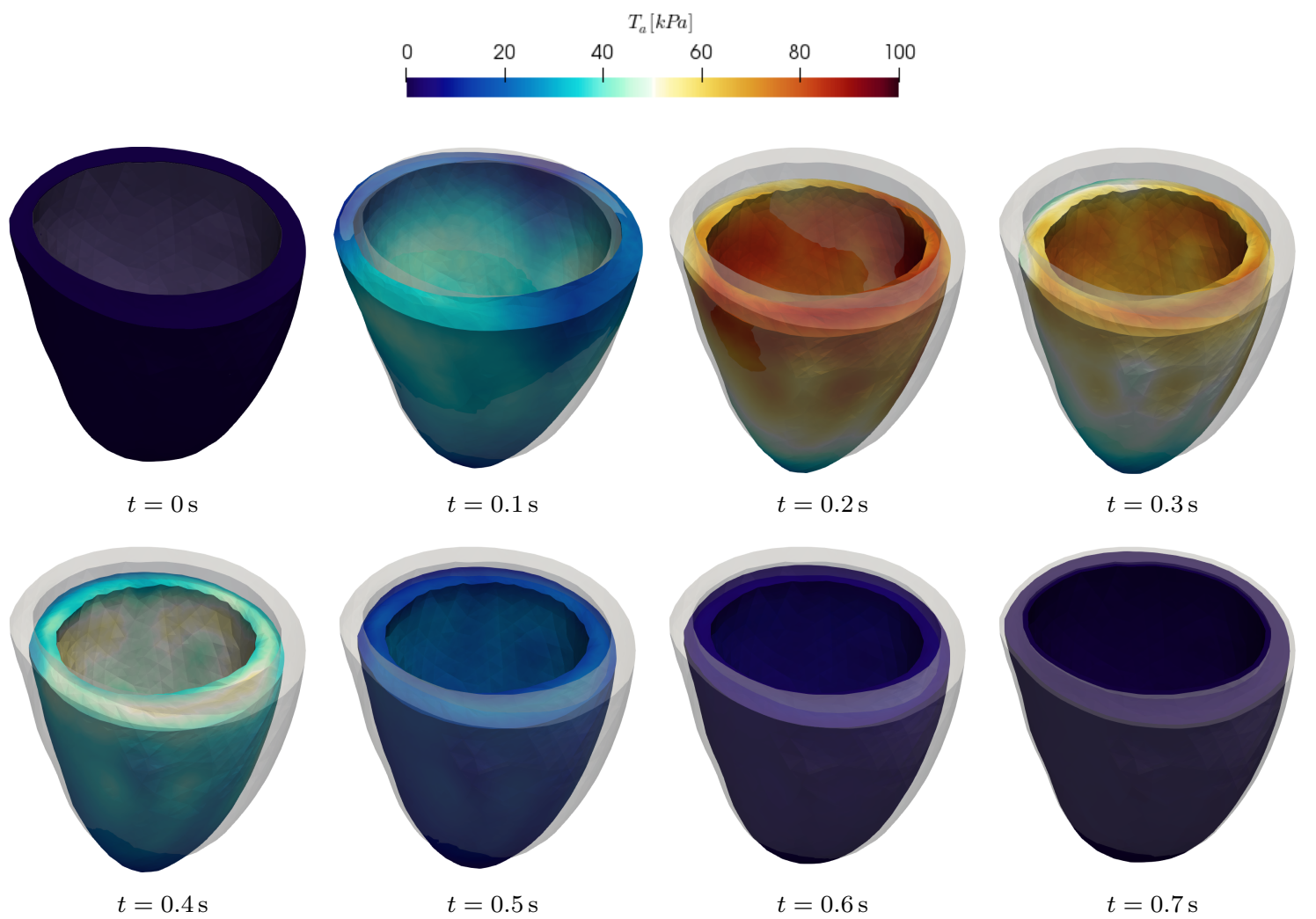

Figure 6: Representation of the variable $T_{\mathrm{a}}$ (active tension) at different times $t$. The variable is represented in the domain in the domain $\Omega(t)$, obtained from the reference domain $\Omega_{0}$ - which is represented in trasparency - by deforming the latter according to the displacement $\mathbf{d}(t)$. 


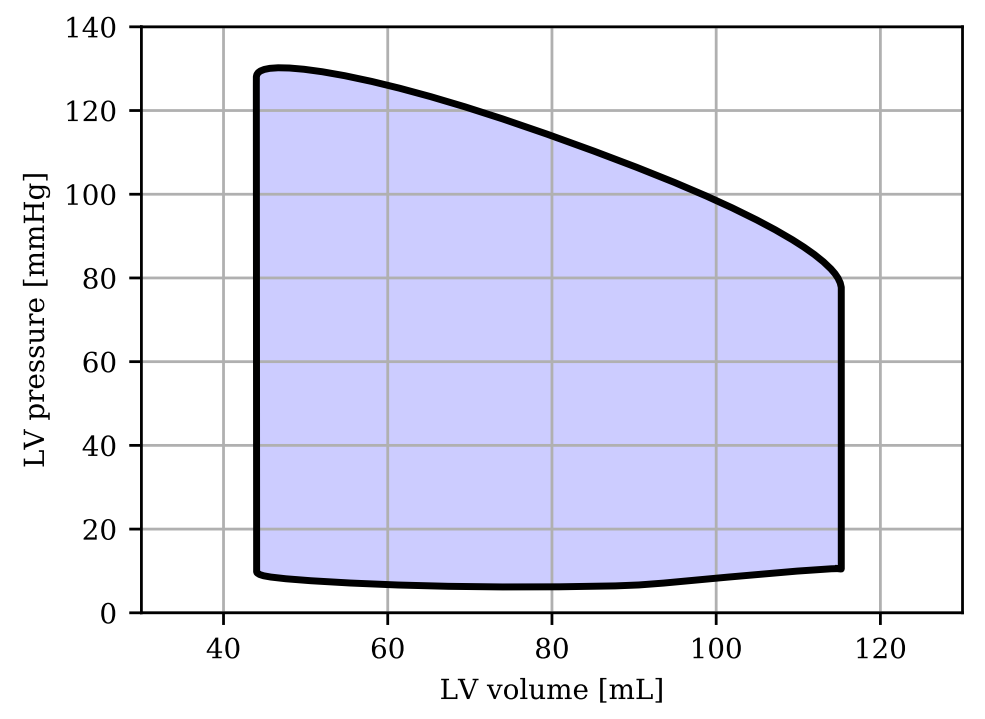

Figure 7: Pressure-volume loop.

and A. Quarteroni have been supported by the Italian research project MIUR PRIN17 2017AXL54F "Modeling the heart across the scales: from cardiac cells to the whole organ". F. Regazzoni has been supported by the Italian research project MIUR PRIN17 2017KL4EF3 "Mathematics of active materials: From mechanobiology to smart devices".
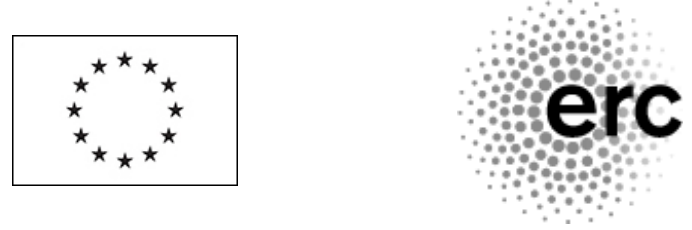

\section{References}

[1] R. R. Aliev and A. V. Panfilov. "A simple two-variable model of cardiac excitation". In: Chaos, Solitons 83 Fractals 7.3 (1996), pp. 293-301.

[2] D. Ambrosi, G. Arioli, F. Nobile, and A. Quarteroni. "Electromechanical Coupling in Cardiac Dynamics: The Active Strain Approach". en. In: SIAM Journal on Applied Mathematics 71.2 (Jan. 2011), pp. 605-621.

[3] D. Ambrosi and S. Pezzuto. "Active Stress vs. Active Strain in Mechanobiology: Constitutive Issues". In: Journal of Elasticity 107.2 (Apr. 2012), pp. 199-212.

[4] L. Antiga, M. Piccinelli, L. Botti, B. Ene-Iordache, A. Remuzzi, and D. A. Steinman. "An image-based modeling framework for patient-specific computational hemodynamics". In: Medical \& biological engineering \& computing 46.11 (2008), p. 1097.

[5] S. S. Antman. Nonlinear Problems of Elasticity. Springer, 1995. 
[6] J. M. Ball. "Convexity conditions and existence theorems in nonlinear elasticity". In: Archive for rational mechanics and Analysis 63.4 (1976), pp. 337-403.

[7] J. D. Bayer, R. C. Blake, G. Plank, and N. A. Trayanova. "A novel rule-based algorithm for assigning myocardial fiber orientation to computational heart models". In: Annals of Biomedical Engineering 40.10 (2012), pp. 2243-2254.

[8] G. W. Beeler and H Reuter. "Reconstruction of the action potential of ventricular myocardial fibres". In: The Journal of physiology 268.1 (1977), pp. 177-210.

[9] D. Bers. Excitation-contraction coupling and cardiac contractile force. Vol. 237. Springer Science \& Business Media, 2001.

[10] P. J. Blanco and R. A. Feijóo. "A 3D-1D-0D Computational Model for the Entire Cardiovascular System". In: Computational Mechanics 24 (2010), pp. 5887-5911.

[11] A. Bueno-Orovio, E. M. Cherry, and F. H. Fenton. "Minimal model for human ventricular action potentials in tissue". In: Journal of Theoretical Biology 253.3 (2008), pp. 544-560.

[12] S. Campbell, F. Lionetti, K. Campbell, and A. McCulloch. "Coupling of adjacent tropomyosins enhances cross-bridge-mediated cooperative activation in a Markov model of the cardiac thin filament". In: Biophysical Journal 98.10 (2010), pp. 2254-2264.

[13] M. Caruel. "Mechanics of Fast Force Recovery in striated muscles". PhD thesis. Ecole Polytechnique, 2011.

[14] M. Caruel, R. Chabiniok, P. Moireau, Y. Lecarpentier, and D. Chapelle. "Dimensional reductions of a cardiac model for effective validation and calibration". In: Biomechanics and Modeling in Mechanobiology 13.4 (2014), pp. 897-914.

[15] M. Caruel, P. Moireau, and D. Chapelle. "Stochastic modeling of chemical-mechanical coupling in striated muscles". In: Biomechanics and Modeling in Mechanobiology 18.3 (2019), pp. 563587.

[16] M. Caruel and L. Truskinovsky. "Physics of muscle contraction". In: Reports on Progress in Physics 81.3 (2018), p. 036602.

[17] B. Cipra. "An introduction to the Ising model". In: American Mathematical Monthly 94.10 (1987), pp. 937-959.

[18] P. Colli Franzone, L. Pavarino, and S. Scacchi. Mathematical cardiac electrophysiology. Vol. 13. Springer, 2014.

[19] B. Dacorogna. Direct methods in the calculus of variations. Vol. 78. Springer Science \& Business Media, 2007.

[20] L. Dedè, A. Gerbi, and A. Quarteroni. "Segregated algorithms for the numerical simulation of cardiac electromechanics in the left human ventricle". In: The Mathematics of Mechanobiology. Ed. by D. Ambrosi and P. Ciarletta. Springer, 2020.

[21] T. Duke. "Molecular model of muscle contraction". In: Proceedings of the National Academy of Sciences 96.6 (1999), pp. 2770-2775.

[22] L. Dupuis, J. Lumens, T. Arts, and T. Delhaas. "Mechano-chemical Interactions in Cardiac Sarcomere Contraction: A Computational Modeling Study". In: PLoS Computational Biology 12.10 (2016), e1005126.

[23] A. Einstein. "Eine neue bestimmung der moleküldimensionen". PhD thesis. ETH Zurich, 1905. 
[24] G. P. Farman, E. J. Allen, K. Q. Schoenfelt, P. H. Backx, and P. P. De Tombe. "The role of thin filament cooperativity in cardiac length-dependent calcium activation". In: Biophysical Journal 99.9 (2010), pp. 2978-2986.

[25] M. Fedele and A. Quarteroni. "Polygonal surface processing and mesh generation tools for the numerical simulation of the cardiac function". In: Int J Numer Meth Biomed Engng (2021).

[26] R. FitzHugh. "Impulses and physiological states in theoretical models of nerve membrane". In: Biophysical Journal 1.6 (1961), pp. 445-466.

[27] D. Fitzsimons, J. Patel, and R. Moss. "Cross-bridge interaction kinetics in rat myocardium are accelerated by strong binding of myosin to the thin filament". In: The Journal of Physiology 530.2 (2001), pp. 263-272.

[28] L. Formaggia, J.-F. Gerbeau, F. Nobile, and A. Quarteroni. "On the coupling of 3D and 1D Navier-Stokes equations for flow problems in compliant vessels". In: Computer methods in applied mechanics and engineering 191.6-7 (2001), pp. 561-582.

[29] L. Formaggia, A. Quarteroni, and A. Veneziani. Cardiovascular Mathematics: Modeling and simulation of the circulatory system. Vol. 1. Springer Science \& Business Media, 2010.

[30] P. C. Franzone and G. Savaré. "Degenerate evolution systems modeling the cardiac electric field at micro-and macroscopic level". In: Evolution equations, semigroups and functional analysis. Springer, 2002, pp. 49-78.

[31] A. Gerbi. "Numerical Approximation of Cardiac Electro-fluid-mechanical Models: Coupling Strategies for Large-scale Simulation". PhD thesis. Ecole Polytechnique Fédérale de Lausanne, 2018.

[32] A. Gerbi, L. Dedè, and A. Quarteroni. "A monolithic algorithm for the simulation of cardiac electromechanics in the human left ventricle". In: Mathematics in Engineering 1.1 (2018), pp. 1-37.

[33] D. B. Geselowitz and W. Miller. "A bidomain model for anisotropic cardiac muscle". In: Annals of biomedical engineering 11.3-4 (1983), pp. 191-206.

[34] J. M. Guccione, K. D. Costa, and A. D. McCulloch. "Finite element stress analysis of left ventricular mechanics in the beating dog heart". In: Journal of Biomechanics 28.10 (1995), pp. $1167-1177$.

[35] J. M. Guccione, A. D. McCulloch, and L. Waldman. "Passive material properties of intact ventricular myocardium determined from a cylindrical model". In: Journal of Biomechanical Engineering 113.1 (1991), pp. 42-55.

[36] D. Heeley, L. Smillie, and E. Lohmeier-Vogel. "Effects of deletion of tropomyosin overlap on regulated actomyosin subfragment 1 ATPase". In: Biochemical Journal 258.3 (1989), pp. 831836.

[37] A. Hill. "The heat of shortening and the dynamic constants of muscle". In: Proceedings of the Royal Society of London B: Biological Sciences 126.843 (1938), pp. 136-195.

[38] A. Hill. "The possible effects of the aggregation of the molecules of haemoglobin on its dissociation curves". In: The Journal of Physiology 40 (1910), pp. 4-7.

[39] M. Hirschvogel, M. Bassilious, L. Jagschies, and et al. "A monolithic 3D-0D coupled closedloop model of the heart and the vascular system: Experiment-based parameter estimation for patient-specific cardiac mechanics". In: International Journal for Numerical Methods in Biomedical Engineering 33.8 (2017), e2842. 
[40] A. L. Hodgkin and A. F. Huxley. "A quantitative description of membrane current and its application to conduction and excitation in nerve". In: The Journal of Physiology 117.4 (1952), pp. 500-544.

[41] G. A. Holzapfel and R. W. Ogden. "Constitutive modelling of passive myocardium: a structurally based framework for material characterization". In: Philosophical Transactions of the Royal Society A: Mathematical, Physical and Engineering Sciences 367.1902 (2009), pp. 34453475 .

[42] P. Hunter, A. McCulloch, and H. Ter Keurs. "Modelling the mechanical properties of cardiac muscle". In: Progress in Biophysics and Molecular Biology 69.2 (1998), pp. 289-331.

[43] J. Hussan, P. de Tombe, and J. Rice. "A spatially detailed myofilament model as a basis for large-scale biological simulations". In: IBM Journal of Research and Development 50.6 (2006), pp. $583-600$.

[44] A. F. Huxley and R. Niedergerke. "Structural Changes in Muscle During Contraction: Interference Microscopy of Living Muscle Fibres". In: Nature 173.4412 (1954), pp. 971-973.

[45] A. Huxley and R. Simmons. "Proposed mechanism of force generation in striated muscle". In: Nature 233.5321 (1971), pp. 533-538.

[46] A. F. Huxley. "Muscle structure and theories of contraction". In: Progress in Biophysics and Biophysical Chemistry 7 (1957), pp. 255-318.

[47] H. Huxley and J. Hanson. "Changes in the Cross-Striations of Muscle during Contraction and Stretch and their Structural Interpretation". In: Nature 173.4412 (1954), pp. 973-976.

[48] G. W. Jenkins, C. P. Kemnitz, and G. J. Tortora. Anatomy and physiology: from science to life. Wiley Hoboken, 2007.

[49] A. M. Katz. Physiology of the heart. Lippincott Williams \& Wilkins, 2010.

[50] J. Keener and J. Sneyd. Mathematical physiology. Vol. 1. Springer, 2009.

[51] F. Kimmig. "Multi-scale modeling of muscle contraction - From stochastic dynamics of molecular motors to continuum mechanics". PhD thesis. Université Paris-Saclay, 2019.

[52] S. Krishnamoorthi, M. Sarkar, and W. S. Klug. "Numerical quadrature and operator splitting in finite element methods for cardiac electrophysiology". In: International journal for numerical methods in biomedical engineering 29.11 (2013), pp. 1243-1266.

[53] S. Land and S. Niederer. "A spatially detailed model of isometric contraction based on competitive binding of troponin I explains cooperative interactions between tropomyosin and crossbridges". In: PLoS Computational Biology 11.8 (2015), e1004376.

[54] S. Land, S. Park-Holohan, N. Smith, C. dos Remedios, J. Kentish, and S. Niederer. "A model of cardiac contraction based on novel measurements of tension development in human cardiomyocytes". In: Journal of Molecular and Cellular Cardiology 106 (2017), pp. 68-83.

[55] S. Land, S. A. Niederer, J. M. Aronsen, E. K. Espe, L. Zhang, W. E. Louch, I. Sjaastad, O. M. Sejersted, and N. P. Smith. "An analysis of deformation-dependent electromechanical coupling in the mouse heart". In: The Journal of Physiology 590.18 (2012), pp. 4553-4569.

[56] C.-h. Luo and Y. Rudy. "A dynamic model of the cardiac ventricular action potential. I. Simulations of ionic currents and concentration changes." In: Circulation research 74.6 (1994), pp. 1071-1096.

[57] C.-h. Luo and Y. Rudy. "A model of the ventricular cardiac action potential. Depolarization, repolarization, and their interaction." In: Circulation research 68.6 (1991), pp. 1501-1526. 
[58] L. Marcucci and L. Truskinovsky. "Mechanics of the power stroke in myosin II". In: Physical Review E 81.5 (2010), p. 051915.

[59] L. Marcucci and L. Truskinovsky. "Muscle contraction: A mechanical perspective". In: The European Physical Journal E 32.4 (2010), pp. 411-418.

[60] C. B. Morrey et al. "Quasi-convexity and the lower semicontinuity of multiple integrals." In: Pacific journal of mathematics 2.1 (1952), pp. 25-53.

[61] S. A. Niederer, P. J. Hunter, and N. P. Smith. "A quantitative analysis of cardiac myocyte relaxation: a simulation study". In: Biophysical Journal 90.5 (2006), pp. 1697-1722.

[62] J. Norris. Markov chains. 2. Cambridge University Press, 1998.

[63] R. W. Ogden. Non-linear elastic deformations. Courier Corporation, 1997.

[64] B. Pan, A. Gordon, and Z. Luo. "Removal of tropomyosin overlap modifies cooperative binding of myosin S-1 to reconstituted thin filaments of rabbit striated muscle." In: Journal of Biological Chemistry 264.15 (1989), pp. 8495-8498.

[65] G. Piazzesi and V. Lombardi. "A cross-bridge model that is able to explain mechanical and energetic properties of shortening muscle." In: Biophysical journal 68.5 (1995), p. 1966.

[66] A. Quarteroni, R. Sacco, and F. Saleri. Numerical mathematics. Vol. 37. Springer Science \& Business Media, 2010.

[67] A. Quarteroni, L. Dede', A. Manzoni, and C. Vergara. Mathematical modelling of the human cardiovascular system: data, numerical approximation, clinical applications. Cambridge Monographs on Applied and Computational Mathematics. Cambridge University Press, 2019.

[68] A. Quarteroni, A. Veneziani, and C. Vergara. "Geometric multiscale modeling of the cardiovascular system, between theory and practice". In: Computer Methods in Applied Mechanics and Engineering 302 (2016), pp. 193-252.

[69] F. Regazzoni, L. Dedè, and A. Quarteroni. "Active contraction of cardiac cells: a reduced model for sarcomere dynamics with cooperative interactions". In: Biomechanics and Modeling in Mechanobiology 17 (2018), pp. 1663-1686.

[70] F. Regazzoni, L. Dedè, and A. Quarteroni. "Active force generation in cardiac muscle cells: mathematical modeling and numerical simulation of the actin-myosin interaction". In: Vietnam Journal of Mathematics (2020), pp. 1-32.

[71] F. Regazzoni, L. Dedè, and A. Quarteroni. "Biophysically detailed mathematical models of multiscale cardiac active mechanics". In: PLOS Computational Biology 16.10 (2020), e1008294.

[72] F. Regazzoni, L. Dedè, and A. Quarteroni. "Machine learning of multiscale active force generation models for the efficient simulation of cardiac electromechanics". In: Computer Methods in Applied Mechanics and Engineering 370 (2020), p. 113268.

[73] F. Regazzoni, M. Salvador, P. C. Africa, M Fedele, L. Dede', and A. Quarteroni. "A cardiac electromechanics model coupled with a lumped parameters model for closed-loop blood circulation. Part I: model derivation". In: arXiv preprint arXiv:2011.15040 (2020).

[74] F. Regazzoni, M. Salvador, P. C. Africa, M Fedele, L. Dede', and A. Quarteroni. "A cardiac electromechanics model coupled with a lumped parameters model for closed-loop blood circulation. Part II: numerical approximation". In: arXiv preprint arXiv:2011.15051 (2020).

[75] F. Regazzoni. "Mathematical modeling and Machine Learning for the numerical simulation of cardiac electromechanics". PhD thesis. Politecnico di Milano, 2020. 
[76] F. Regazzoni and A. Quarteroni. "An oscillation-free fully partitioned scheme for the numerical modeling of cardiac active mechanics". In: Computer Methods in Applied Mechanics and Engineering 373 (2021), p. 113506.

[77] J. Rice, G. Stolovitzky, Y. Tu, and P. de Tombe. "Ising model of cardiac thin filament activation with nearest-neighbor cooperative interactions". In: Biophysical Journal 84.2 (2003), pp. 897909.

[78] J. Rice and P. de Tombe. "Approaches to modeling crossbridges and calcium-dependent activation in cardiac muscle". In: Progress in Biophysics and Molecular Biology 85.2 (2004), pp. 179-195.

[79] J. Rice, F. Wang, D. Bers, and P. de Tombe. "Approximate model of cooperative activation and crossbridge cycling in cardiac muscle using ordinary differential equations". In: Biophysical Journal 95.5 (2008), pp. 2368-2390.

[80] J. Rice, R. Winslow, and W. Hunter. "Comparison of putative cooperative mechanisms in cardiac muscle: length dependence and dynamic responses". In: American Journal of PhysiologyHeart and Circulatory Physiology 276.5 (1999), H1734-H1754.

[81] O. H. Schmitt. "Biological information processing using the concept of interpenetrating domains". In: Information processing in the nervous system. Springer, 1969, pp. 325-331.

[82] V. Sequeira and J. van der Velden. "The Frank-Starling Law: a jigsaw of titin proportions". In: Biophysical Reviews 9.3 (2017), pp. 259-267.

[83] D. Smith, M. A. Geeves, J Sleep, and S. M. Mijailovich. "Towards a unified theory of muscle contraction. I: foundations". In: Annals of Biomedical Engineering 36.10 (2008), pp. 16241640.

[84] Y.-B. Sun, F. Lou, and M. Irving. "Calcium-and myosin-dependent changes in troponin structure during activation of heart muscle". In: The Journal of Physiology 587.1 (2009), pp. 155163.

[85] A. Tagliabue, L. Dede', and A. Quarteroni. "Complex blood flow patterns in an idealized left ventricle: a numerical study". In: Chaos: an Interdisciplinary Journal of Nonlinear Science 27.9 (2017), p. 093939.

[86] A. Tagliabue, L. Dede', and A. Quarteroni. "Fluid dynamics of an idealized left ventricle: the extended Nitsche's method for the treatment of heart valves as mixed time varying boundary conditions". In: International Journal for Numerical Methods in Fluids 85.3 (2017), pp. 135164 .

[87] K. Ten Tusscher, D. Noble, P.-J. Noble, and A. V. Panfilov. "A model for human ventricular tissue". In: American Journal of Physiology-Heart and Circulatory Physiology 286.4 (2004), H1573-H1589.

[88] K. H. Ten Tusscher and A. V. Panfilov. "Alternans and spiral breakup in a human ventricular tissue model". In: American Journal of Physiology-Heart and Circulatory Physiology 291.3 (2006), H1088-H1100.

[89] J. Tomek, A. Bueno-Orovio, E. Passini, X. Zhou, A. Minchole, O. Britton, C. Bartolucci, S. Severi, A. Shrier, L. Virag, et al. "Development, calibration, and validation of a novel human ventricular myocyte model in health, disease, and drug block". In: Elife 8 (2019), e48890.

[90] G. J. Tortora and B. H. Derrickson. Principles of anatomy and physiology. John Wiley \& Sons, 2008. 
[91] T. P. Usyk, I. J. LeGrice, and A. D. McCulloch. "Computational model of three-dimensional cardiac electromechanics". In: Computing and Visualization in Science 4.4 (2002), pp. 249257.

[92] M. Veneroni. "Reaction-diffusion systems for the macroscopic bidomain model of the cardiac electric field". In: Nonlinear Analysis: Real World Applications 10.2 (2009), pp. 849-868.

[93] T. Washio, J. Okada, S. Sugiura, and T. Hisada. "Approximation for cooperative interactions of a spatially-detailed cardiac sarcomere model". In: Cellular and Molecular Bioengineering 5.1 (2012), pp. 113-126.

[94] T. Washio, J. Okada, A. Takahashi, K. Yoneda, Y. Kadooka, S. Sugiura, and T. Hisada. "Multiscale heart simulation with cooperative stochastic cross-bridge dynamics and cellular structures". In: Multiscale Modeling \& Simulation 11.4 (2013), pp. 965-999.

[95] T. Washio, K. Yoneda, J. Okada, T. Kariya, S. Sugiura, and T. Hisada. "Ventricular fiber optimization utilizing the branching structure". In: International Journal for Numerical Methods in Biomedical Engineering (2015), e02753.

[96] Zygote 3D models. 2019. URL: https://www.zygote.com/. 